\title{
STOCHASTIC BURGERS' EQUATION ON THE REAL LINE: REGULARITY AND MOMENT ESTIMATES
}

\author{
PETER LEWIS AND DAVID NUALART*
}

\begin{abstract}
In this project we investigate the stochastic Burgers' equation with multiplicative space-time white noise on an unbounded spatial domain. We give a random field solution to this equation by defining a process via a kind of Feynman-Kac representation which solves a stochastic partial differential equation such that its Hopf-Cole transformation solves Burgers' equation. Finally, we obtain Hölder regularity and moment estimates for the solution to Burgers' equation.
\end{abstract}

\section{INTRODUCTION}

We are concerned with the following (formal) version of Burgers' equation

$$
\frac{\partial}{\partial t} u(t, x)=\frac{\partial^{2}}{\partial x^{2}} u(t, x)-\frac{1}{2} \frac{\partial}{\partial x} u(t, x)^{2}+\sigma(t, x, u(t, x)) \frac{\partial^{2} W}{\partial t \partial x}
$$

indexed by $(t, x) \in[0, T] \times \mathbb{R}$, given a nonrandom initial condition $u_{0}$ and a Brownian sheet $W$. To study this equation rigorously, we understand the above in its mild form; that is, as an integral equation:

$$
\begin{aligned}
u(t, x) & =\int_{\mathbb{R}} G_{t}(x-y) u_{0}(y) d y+\frac{1}{2} \int_{0}^{t} \int_{\mathbb{R}} \frac{\partial}{\partial y} G_{t-s}(x-y) u(s, y)^{2} d y d s \\
& +\int_{0}^{t} \int_{\mathbb{R}} G_{t-s}(x-y) \sigma_{s}(y) W(d s, d y),
\end{aligned}
$$

where $\sigma_{t}(x) \equiv \sigma(t, x, u(t, x))$ is used for shorthand, $G$ is the heat kernel

$$
G_{t}(x)=(4 \pi t)^{-1 / 2} e^{-x^{2} / 4 t},
$$

and the stochastic integral is understood in the Walsh sense.

In [1], the authors investigate this equation with $\sigma \equiv 1$; that is, with additive noise. In [4], multiplicative noise ( $\sigma$ depending on $u$ ) is studied on the spatial interval $[0,1]$, rather than on $\mathbb{R}$. The authors of both of these papers construct an explicit solution by defining a process via a Feynman-Kac representation such that its Hopf-Cole transformation solves Burgers' equation. On the other hand, existence and uniqueness to a general class of semilinear stochastic partial differential equations (SPDEs) on unbounded spatial domains, which contains (1.1), is shown by Gyöngy and Nualart in [3] using fixed point arguments which follow from some maximal inequalities on stochastic convolutions. However, they do not consider an explicit construction of the solution. Hence, the primary aim of this paper is to

2010 Mathematics Subject Classification. Primary 60H15. Secondary 60H20, 60 G60.

Key words and phrases. Stochastic Burgers' equation, Feynman-Kac representation, space-time white noise.

*David Nualart is partially supported by the NSF grant DMS1512891. 
construct a solution to (1.1), similar to what is done in [1] and [4], by defining and transforming a process with a Feynman-Kac representation. We then obtain uniqueness for free from [3]. Finally, we prove Hölder regularity and moment estimates to the solution of Burgers' equation. In the case of additive noise, such moment estimates have been studied. However, to our knowledge, Hölder regularity has not been established in other works on stochastic Burgers' equation with space-time white noise.

The paper is organized as follows. First, we define a process, $\psi$, via a kind of FeynmanKac representation. Then, we establish several properties of $\psi$, such as moment bounds, Hölder regularity, and differentiability. Next, we show that the Hopf-Cole transformation of $\psi$, which (formally at the moment) is

$$
u(t, x)=-2 \frac{\partial}{\partial x} \log \psi(t, x),
$$

solves (1.1). Appealing to the uniqueness result in [3], our solution is unique. Lastly, we obtain Hölder regularity and an upper bound on moments of the solution to Burgers' equation using properties of the process $\psi$.

Throughout much of the paper, we follow similar steps as in [4], but have to adjust almost all of the arguments to handle the challenges posed by an unbounded domain. As such, due to difficulties with integrability, many of our assumptions differ from those in [4], though they are consistent with [3].

\section{PRELiminaries}

Let $W=\left\{W(t, x), t \in \mathbb{R}_{+}, x \in \mathbb{R}\right\}$ be a zero-mean Gaussian random field defined on a complete probability space $(\Omega, \mathcal{F}, \mathbb{P})$, with covariance

$$
\mathbb{E}[W(s, x) W(t, y)]=(s \wedge t)(|x| \wedge|y|) \mathbf{1}_{[0, \infty)}(x y)
$$

for $s, t \geq 0, x, y \in \mathbb{R}$. In other words, $W$ is a Brownian sheet on $\mathbb{R}^{2}$. For any $t \geq 0$, we denote by $\mathcal{F}_{t}$ the $\sigma$-field generated by the random variables $\{W(s, x), s \in[0, t], x \in \mathbb{R}\}$ and the sets of probability zero. The stochastic integral with respect to $W$ in (1.1) is understood in the Walsh sense. For a careful treatment of this integration theory, see John Walsh's seminal work on SPDEs [5], for example. We use the notation $\mathbb{E}(\cdot)$ to represent expectation with respect to $W$, and denote its corresponding norm by $\|\cdot\|_{p}=\mathbb{E}\left(|\cdot|^{p}\right)^{1 / p}$. We will make use of the convention $\mathbf{1}_{[b, a]}=-\mathbf{1}_{[a, b]}$, whenever $b>a$.

Throughout the paper we assume the following conditions:

(A1) The initial condition $u_{0}$ is a deterministic, continuous, and bounded function such that $u_{0} \in L^{2}(\mathbb{R}) \cap L^{1}(\mathbb{R})$.

(A2) $\sigma: \mathbb{R}_{+} \times \mathbb{R}^{2} \rightarrow \mathbb{R}$ is a Borel function satisfying the following Lipschitz and growth properties

$$
\begin{aligned}
|\sigma(t, x, r)-\sigma(t, x, v)| & \leq L|r-v| \\
|\sigma(t, x, r)| & \leq f(x)
\end{aligned}
$$

for all $t \geq 0, x, r, v \in \mathbb{R}$ and for some constant $L>0$ and some non-negative function $f \in L^{2}(\mathbb{R}) \cap L^{q}(\mathbb{R})$, where $q>2$. 
Under these conditions, it is proved by Gyöngy and Nualart in [3] that there exists a unique $L^{2}(\mathbb{R})$-valued $\mathcal{F}_{t}$-adapted continuous stochastic process $u=\{u(t), t \geq 0\}$, which satisfies the integral equation (1.1). Furthermore, the process $u$ has a continuous version in $(t, x)$.

Before our discussion of the Feynman-Kac representation, we prove a technical lemma regarding regularity of the heat kernel $G_{t}(x)=(4 \pi t)^{-1 / 2} e^{-x^{2} / 4 t}$ that will be used several times in the paper.

Lemma 2.1. Let $\theta_{1}>0, \theta_{2} \geq 0$ and $\beta>0$ be such that

$$
\beta\left(\theta_{1}-\theta_{2}-1\right)<2<\beta\left(3 \theta_{1}-\theta_{2}-1\right) .
$$

Then, for any $0<t_{1}<t_{2}$, we have

$$
\int_{0}^{t_{1}}\left(\int_{\mathbb{R}}\left|G_{t_{2}-s}(x)-G_{t_{1}-s}(x)\right|^{\theta_{1}}|x|^{\theta_{2}} d x\right)^{\beta} d s \leq C\left(t_{2}-t_{1}\right)^{1-\beta\left(\theta_{1}-\theta_{2}-1\right) / 2},
$$

for some constant $C$ depending on $\theta_{1}, \theta_{2}$ and $\beta$.

Proof. Set $\tau=t_{2}-t_{1}$. Making the change of variables $x=\sqrt{s} y$ and $s=\tau / \sigma$, yields

$$
\begin{gathered}
\int_{0}^{t_{1}}\left(\int_{\mathbb{R}}\left|G_{t_{2}-s}(x)-G_{t_{1}-s}(x)\right|^{\theta_{1}}|x|^{\theta_{2}} d x\right)^{\beta} d s \\
=\int_{0}^{t_{1}}\left(\int_{\mathbb{R}}\left|\frac{1}{\sqrt{4 \pi(\tau+s)}} e^{-\frac{x^{2}}{4(\tau+s)}}-\frac{1}{\sqrt{4 \pi s}} e^{-\frac{x^{2}}{4 s}}\right|^{\theta_{1}}|x|^{\theta_{2}} d x\right)^{\beta} d s \\
\leq C\left(t_{2}-t_{1}\right)^{1-\beta\left(\theta_{1}-\theta_{2}-1\right) / 2} \int_{0}^{\infty} \sigma^{-2+\beta\left(\theta_{1}-\theta_{2}-1\right) / 2} \\
\times\left(\int_{\mathbb{R}}\left|\frac{1}{\sqrt{\sigma+1}} e^{-\frac{y^{2}}{4(\sigma+1)}}-e^{-\frac{y^{2}}{4}}\right|^{\theta_{1}}|y|^{\theta_{2}} d y\right)^{\beta} d \sigma .
\end{gathered}
$$

Then, condition (2.3) implies that the above integral in $d \sigma$ is finite, and we get the desired estimate.

Throughout the paper we will denote by $C$ a generic constant that might depend on $\sigma, f$, $u_{0}, T$ and the exponent $p$ we are considering. The value of this constant may be different from line to line. However, we will specify dependence where we feel it may be relevant.

\section{Feynman-Kac Representation}

We now define a process via a kind of Feynman-Kac formula that will be the main focus of this paper. Given $u_{0}$, set

$$
\psi_{0}(x):=\exp \left\{-\frac{1}{2} \int_{0}^{x} u_{0}(y) d y\right\}
$$

with the convention that the integral is on the interval $[x, 0]$ if $x<0$. Let $\beta=\left\{\beta_{s}, s \in[0, t]\right\}$ be a backward Brownian motion (BWBM) that is independent of $W$, starting at $x \in \mathbb{R}$ at time $t$ and with variance $2(t-s)$. We use the notation $\mathbb{E}_{x, t}^{\beta}$ to denote the expectation with 
respect to the law of the BWBM. Let $u$ be the mild solution to Burgers' equation. That is, $u$ satisfies (1.1). We will make use of the notation $\sigma_{s}(y):=\sigma(s, y, u(s, y))$. Set

$$
M_{t}^{\beta}:=\int_{0}^{t} \int_{\mathbb{R}} \sigma_{s}(y) \mathbf{1}_{\left[0, \beta_{s}\right]}(y) W(d s, d y),
$$

with the convention that the indicator function is on the interval $\left[\beta_{s}, 0\right]$ if the $\mathrm{BWBM}$ is negative at time $s$. Observe that this stochastic integral is a well-defined martingale due to the square-integrability assumption (2.2) on $\sigma$. With the above notation in mind, define the two-parameter stochastic process $\psi$ by

$$
\psi(t, x):=\mathbb{E}_{x, t}^{\beta}\left[\psi_{0}\left(\beta_{0}\right) e^{-\frac{1}{2} M_{t}^{\beta}}\right] .
$$

We first establish some estimates of moments of the process $\psi$, then show that it satisfies a certain integral equation.

Proposition 3.1. For all $t \geq 0, x \in \mathbb{R}$, and integers $p \geq 2$, we have moment estimates of the form

$$
\|\psi(t, x)\|_{p} \leq \exp \left(\frac{t p}{4}\|f\|_{L^{2}(\mathbb{R})}^{2}+\frac{1}{2}\left\|u_{0}\right\|_{L^{1}(\mathbb{R})}\right)
$$

Proof. Let $\vec{\beta}=\left\{\beta^{i}\right\}_{i=1}^{p}$ be $p$ independent backward Brownian motions on $[0, t]$ starting at $x \in \mathbb{R}$ at time $t$, with variance $2(t-s)$. By independence and Fubini's theorem, we have

$$
\begin{aligned}
\|\psi(t, x)\|_{p}^{p}=\mathbb{E}\left(|\psi(t, x)|^{p}\right) & =\mathbb{E}\left[\prod_{i=1}^{p} \mathbb{E}_{x, t}^{\beta^{i}}\left(\psi_{0}\left(\beta_{0}^{i}\right) e^{-\frac{1}{2} M_{t}^{\beta^{i}}}\right)\right] \\
& =\mathbb{E}\left[\mathbb{E}_{x, t}^{\vec{\beta}}\left(\prod_{i=1}^{p} \psi_{0}\left(\beta_{0}^{i}\right) e^{-\frac{1}{2} M_{t}^{\beta^{i}}}\right)\right] \\
& =\mathbb{E}_{x, t}^{\vec{\beta}}\left[\left(\prod_{i=1}^{p} \psi_{0}\left(\beta_{0}^{i}\right)\right) \mathbb{E}\left(\exp \left\{-\frac{1}{2} \sum_{j=1}^{p} M_{t}^{\beta^{j}}\right\}\right)\right] .
\end{aligned}
$$

Now, by the multivariate Itô formula,

$$
e^{-\frac{1}{2} \sum_{j=1}^{p} M_{t}^{\beta^{j}}}=1-\frac{1}{2} \int_{0}^{t} \sum_{i=1}^{p} e^{-\frac{1}{2} \sum_{j=1}^{p} M_{s}^{\beta^{j}}} d M_{s}^{\beta^{i}}+\int_{0}^{t} \sum_{i, j=1}^{p} \frac{1}{8} e^{-\frac{1}{2} \sum_{k=1}^{p} M_{s}^{\beta^{k}}} d\left\langle M^{\beta^{i}}, M^{\beta^{j}}\right\rangle_{s} .
$$

Since the quadratic covariation of these martingales is

$$
\left\langle M^{\beta^{i}}, M^{\beta^{j}}\right\rangle_{t}=\int_{0}^{t} d s \int_{\mathbb{R}} d y \sigma_{s}^{2}(y) \mathbf{1}_{\left[0, \beta_{s}^{i}\right]}(y) \mathbf{1}_{\left[0, \beta_{s}^{j}\right]}(y),
$$

taking the expectation of the above Itô expansion yields

$$
\begin{aligned}
\mathbb{E}\left(e^{-\frac{1}{2} \sum_{j=1}^{p} M_{t}^{\beta^{j}}}\right) & =1+\frac{1}{8} \int_{0}^{t} \mathbb{E}\left(e^{-\frac{1}{2} \sum_{k=1}^{p} M_{s}^{\beta^{k}}} \sum_{i, j=1}^{p} \mathbf{1}_{\beta_{s}^{i} \beta_{s}^{j}>0} \int_{0}^{\left|\beta^{i}\right| \wedge\left|\beta^{j}\right|}\left[\sigma_{s}^{2}(y)+\sigma_{s}^{2}(-y)\right] d y\right) d s \\
& \leq 1+\frac{p^{2}}{4}\|f\|_{L^{2}(\mathbb{R})}^{2} \int_{0}^{t} \mathbb{E}\left(e^{-\frac{1}{2} \sum_{j=1}^{p} M_{s}^{\beta^{j}}}\right) d s .
\end{aligned}
$$


Recall a version of Gronwall's lemma which states that if a function $g$ satisfies $g(t) \leq$ $a(t)+\int_{0}^{t} b(s) g(s) d s$, where $a$ is non-decreasing and $b$ is non-negative, then $g$ satisfies $g(t) \leq$ $a(t) e^{\int_{0}^{t} b(s) d s}$. Hence, we have

$$
\mathbb{E}\left(e^{-\frac{1}{2} \sum_{j=1}^{p} M_{t}^{\beta^{j}}}\right) \leq \exp \left(\frac{\|f\|_{L^{2}(\mathbb{R})}^{2}}{4} t p^{2}\right)
$$

Therefore,

$$
\|\psi(t, x)\|_{p}^{p}=\mathbb{E}_{x, t}^{\vec{\beta}}\left[\left(\prod_{i=1}^{p} \psi_{0}\left(\beta_{0}^{i}\right)\right) \mathbb{E}\left(\exp \left\{-\frac{1}{2} \sum_{j=1}^{p} M_{t}^{\beta^{j}}\right\}\right)\right] \leq a^{p} e^{b t p^{2}},
$$

where $a=e^{\frac{1}{2}\left\|u_{0}\right\|_{L^{1}(\mathbb{R})}}$ and $b=\frac{1}{4}\|f\|_{L^{2}(\mathbb{R})}^{2}$.

Remark 3.2. Using Jensen's inequality we can show, in the same way as before, that for all integers $p \geq 2$,

$$
\left\|\psi(t, x)^{-1}\right\|_{p}^{p} \leq \exp \left(\frac{t p}{8}\|f\|_{L^{2}(\mathbb{R})}^{2}+\frac{1}{2}\left\|u_{0}\right\|_{L^{1}(\mathbb{R})}\right)
$$

In fact,

$$
\psi(t, x)^{-1} \leq \mathbb{E}_{x, t}^{\beta}\left[\exp \left\{\frac{1}{2} \int_{0}^{x} u_{0}(y) d y+\frac{1}{2} M_{t}^{\beta}\right\}\right] .
$$

Proposition 3.1 implies that for any $T>0$

$$
M_{p, T}:=\sup _{t \in[0, T], x \in \mathbb{R}}\|\psi(t, x)\|_{p}<\infty
$$

and

$$
\sup _{t \in[0, T], x \in \mathbb{R}}\left\|\psi(t, x)^{-1}\right\|_{p}<\infty
$$

for all real numbers $p \geq 2$.

Next, we show that $\psi$ satisfies a particular integral equation.

Proposition 3.3. Let $\psi$ be the process defined in (3.1) and let $G_{t}(x)$ be the heat kernel as before. Then, for $t \geq 0, x \in \mathbb{R}, \psi(t, x)$ satisfies

$$
\begin{aligned}
\psi(t, x) & =\int_{\mathbb{R}} G_{t}(x-y) \psi_{0}(y) d y-\frac{1}{2} \int_{0}^{t} \int_{S} \operatorname{sign}(y) G_{t-s}(x-z) \psi(s, z) \sigma_{s}(y) d z W(d s, d y) \\
& +\frac{1}{8} \int_{0}^{t} \int_{S} G_{t-s}(x-z) \psi(s, z) \sigma_{s}^{2}(y) d z d y d s
\end{aligned}
$$

where

$$
S:=\left\{(y, z) \in \mathbb{R}^{2}:|z| \geq|y|, \text { and } y z \geq 0\right\} .
$$

Proof. The proof of this result follows from the same arguments as in [4]. We briefly explain the main idea. First, observe that $\beta_{0}$ satisfies $\mathbb{E}_{x, t}^{\beta}\left(\psi_{0}\left(\beta_{0}\right)\right)=\int_{\mathbb{R}} G_{t}(x-y) \psi_{0}(y) d y$ since 
$y \mapsto G_{t}(x-y)$ is the density of $\beta_{0}$. Now, apply Itô's formula to get

$$
e^{-\frac{1}{2} M_{t}^{\beta}}=1-\frac{1}{2} \int_{0}^{t} \int_{\mathbb{R}} e^{-\frac{1}{2} M_{s}^{\beta}} \sigma_{s}(y) \mathbf{1}_{\left[0, \beta_{s}\right]}(y) W(d s, d y)+\frac{1}{8} \int_{0}^{t} \int_{\mathbb{R}} e^{-\frac{1}{2} M_{s}^{\beta}} \sigma_{s}(y)^{2}\left|\mathbf{1}_{\left[0, \beta_{s}\right]}(y)\right| d y d s .
$$

Multiply by $\psi_{0}\left(\beta_{0}\right)$ and take the expectation with respect to the BWBM to see that

$$
\begin{aligned}
\psi(t, x)=\int_{\mathbb{R}} G_{t}(x-y) \psi_{0}(y) d y- & \frac{1}{2} \int_{0}^{t} \int_{\mathbb{R}} \sigma_{s}(y) \mathbb{E}_{x, t}^{\beta}\left(\psi_{0}\left(\beta_{0}\right) e^{-\frac{1}{2} M_{s}^{\beta}} \mathbf{1}_{\left[0, \beta_{s}\right]}(y)\right) W(d s, d y) \\
& +\frac{1}{8} \int_{0}^{t} \int_{\mathbb{R}} \sigma_{s}(y)^{2} \mathbb{E}_{x, t}^{\beta}\left(\psi_{0}\left(\beta_{0}\right) e^{-\frac{1}{2} M_{s}^{\beta}}\left|\mathbf{1}_{\left[0, \beta_{s}\right]}(y)\right|\right) d y d s .
\end{aligned}
$$

Finally, apply the Markov property to get

$$
\begin{aligned}
\mathbb{E}_{x, t}^{\beta}\left(\psi_{0}\left(\beta_{0}\right) e^{-\frac{1}{2} M_{s}^{\beta}} \mathbf{1}_{\left[0, \beta_{s}\right]}(y)\right) & =\mathbb{E}_{x, t}^{\beta}\left[\mathbb{E}\left(\psi_{0}\left(\beta_{0}\right) e^{-\frac{1}{2} M_{s}^{\beta}} \mathbf{1}_{\left[0, \beta_{s}\right]}(y) \mid \beta_{r}, s \leq r \leq t\right)\right] \\
& =\mathbb{E}_{x, t}^{\beta}\left[\mathbf{1}_{\left[0, \beta_{s}\right]}(y) \mathbb{E}\left(\psi_{0}\left(\beta_{0}\right) e^{-\frac{1}{2} M_{s}^{\beta}} \mid \beta_{s}\right)\right] \\
& =\int_{0}^{\infty} G_{t-s}(x-z) \mathbb{E}_{z, s}^{\beta}\left(\psi_{0}\left(\beta_{0}\right) e^{-\frac{1}{2} M_{s}^{\beta}}\right) \mathbf{1}_{[0, z]}(y) d z \\
& = \begin{cases}\int_{y}^{\infty} G_{t-s}(x-z) \psi(s, z) d z & \text { if } y \geq 0 \\
-\int_{-\infty}^{y} G_{t-s}(x-z) \psi(s, z) d z & \text { if } y<0 .\end{cases}
\end{aligned}
$$

Similarly,

$$
\mathbb{E}_{x, t}^{\beta}\left(\psi_{0}\left(\beta_{0}\right) e^{-\frac{1}{2} M_{s}^{\beta}}\left|\mathbf{1}_{\left[0, \beta_{s}\right]}(y)\right|\right)= \begin{cases}\int_{y}^{\infty} G_{t-s}(x-z) \psi(s, z) d z & \text { if } y \geq 0 \\ \int_{-\infty}^{y} G_{t-s}(x-z) \psi(s, z) d z & \text { if } y<0 .\end{cases}
$$

Hence, we have the desired result.

Next, we establish a Hölder regularity property for $\psi$.

Proposition 3.4. For $p \geq 2$ and $T>0$, there exists some constant $C$, depending on $p, T$, $\left\|u_{0}\right\|_{\infty},\left\|u_{0}\right\|_{L^{1}(\mathbb{R})}$, and $\|f\|_{L^{2}(\mathbb{R})}$, such that for all $s, t \in[0, T]$, and $x, y \in \mathbb{R}$,

$$
\|\psi(t, x)-\psi(s, y)\|_{p} \leq C\left(|t-s|^{1 / 2}+|x-y|^{1 / 2}\right) .
$$

Proof. First we prove the Hölder continuity in the space variable. Let $x_{1}$ and $x_{2}$ be such that $\left|x_{1}-x_{2}\right|=\delta$. Because $\|\psi(t, x)\|_{p}$ is uniformly bounded on $[0, T] \times \mathbb{R}$, we can assume 
that $\delta \leq 1$. We have

$$
\begin{aligned}
\psi( & \left(, x_{1}\right)-\psi\left(t, x_{2}\right) \\
= & \int_{\mathbb{R}}\left[G_{t}\left(x_{1}-y\right)-G_{t}\left(x_{2}-y\right)\right] \psi_{0}(y) d y \\
& -\frac{1}{2} \int_{0}^{t} \int_{S} \operatorname{sign}(y)\left[G_{t-s}\left(x_{1}-z\right)-G_{t-s}\left(x_{2}-z\right)\right] \psi(s, z) \sigma_{s}(y) d z W(d s, d y) \\
& +\frac{1}{8} \int_{0}^{t} \int_{S}\left[G_{t-s}\left(x_{1}-z\right)-G_{t-s}\left(x_{2}-z\right)\right] \psi(s, z) \sigma_{s}^{2}(y) d z d y d s \\
= & : I_{1}\left(x_{1}, x_{2}\right)-\frac{1}{2} I_{2}\left(x_{1}, x_{2}\right)+\frac{1}{8} I_{3}\left(x_{1}, x_{2}\right) .
\end{aligned}
$$

We make a change of variables to get

$$
\left|I_{1}\left(x_{1}, x_{2}\right)\right| \leq \int_{\mathbb{R}} G_{t}(u)\left|\psi_{0}\left(x_{1}-u\right)-\psi_{0}\left(x_{2}-u\right)\right| d u .
$$

By Hypothesis (A1) the function $\psi_{0}$ has a bounded derivative:

$$
\left|\psi^{\prime}(x)\right| \leq\left\|u_{0}\right\|_{\infty} e^{\frac{1}{2}\left\|u_{0}\right\|_{L^{1}(\mathbb{R})}}
$$

Therefore, it is Lipschitz and we obtain

$$
\left|I_{1}\left(x_{1}, x_{2}\right)\right| \leq C\left|x_{1}-x_{2}\right|=C \delta
$$

Consider the decomposition

$$
I_{2}\left(x_{1}, x_{2}\right)=I_{2,+}\left(x_{1}, x_{2}\right)+I_{2,-}\left(x_{1}, x_{2}\right)
$$

where

$$
I_{2,+}\left(x_{1}, x_{2}\right)=\int_{0}^{t} \int_{0}^{\infty} \sigma_{s}(y) \int_{y}^{\infty}\left[G_{t-s}\left(x_{1}-z\right)-G_{t-s}\left(x_{2}-z\right)\right] \psi(s, z) d z W(d s, d y)
$$

and

$$
I_{2,-}\left(x_{1}, x_{2}\right)=-\int_{0}^{t} \int_{-\infty}^{0} \sigma_{s}(y) \int_{-\infty}^{y}\left[G_{t-s}\left(x_{1}-z\right)-G_{t-s}\left(x_{2}-z\right)\right] \psi(s, z) d z W(d s, d y) .
$$

Applying Burkholder's and Minkowski's inequalities, we get

$$
\begin{aligned}
& \left\|I_{2,+}\left(x_{1}, x_{2}\right)\right\|_{p} \\
& \quad \leq c_{p}\left\|\int_{0}^{t} \int_{0}^{\infty} \sigma_{s}^{2}(y)\left(\int_{y}^{\infty} \psi(s, z)\left[G_{t-s}\left(x_{1}-z\right)-G_{t-s}\left(x_{2}-z\right)\right] d z\right)^{2} d y d s\right\|_{p / 2}^{1 / 2} \\
& \quad \leq c_{p}\left(\int_{0}^{t} \int_{0}^{\infty} f^{2}(y)\left\|\int_{y}^{\infty} \psi(s, z)\left[G_{t-s}\left(x_{1}-z\right)-G_{t-s}\left(x_{2}-z\right)\right] d z\right\|_{p}^{2} d y d s\right)^{1 / 2} .
\end{aligned}
$$


Making a change of variables we can write

$$
\begin{aligned}
\int_{y}^{\infty} & \psi(s, z)\left[G_{t-s}\left(x_{1}-z\right)-G_{t-s}\left(x_{2}-z\right)\right] d z \\
& =\int_{-\infty}^{x_{1}-y} \psi\left(s, x_{1}-u\right) G_{t-s}(u) d u-\int_{-\infty}^{x_{2}-y} \psi\left(s, x_{2}-u\right) G_{t-s}(u) d u \\
& =\int_{-\infty}^{x_{1}-y}\left[\psi\left(s, x_{1}-u\right)-\psi\left(x_{2}-u\right)\right] G_{t-s}(u) d u+\int_{x_{2}-y}^{x_{1}-y} \psi\left(s, x_{2}-u\right) G_{t-s}(u) d u .
\end{aligned}
$$

This leads to the estimate

$$
\begin{aligned}
& \left\|\int_{y}^{\infty} \psi(s, z)\left[G_{t-s}\left(x_{1}-z\right)-G_{t-s}\left(x_{2}-z\right)\right] d z\right\|_{p} \\
& \quad \leq \int_{\mathbb{R}}\left\|\psi\left(s, x_{1}-u\right)-\psi\left(s, x_{2}-u\right)\right\|_{p} G_{t-s}(u) d u+\int_{x_{2}-y}^{x_{1}-y}\left\|\psi\left(s, x_{2}-u\right)\right\|_{p} G_{t-s}(u) d u .
\end{aligned}
$$

Let $M_{p, T}$ be the constant introduced in (3.4) and set

$$
V_{s}:=\sup _{|x-y|=\delta}\|\psi(s, x)-\psi(s, y)\|_{p} .
$$

Then, by Cauchy-Schwarz inequality,

$$
\begin{aligned}
\left\|\int_{y}^{\infty} \psi(s, z)\left[G_{t-s}\left(x_{1}-z\right)-G_{t-s}\left(x_{2}-z\right)\right] d z\right\|_{p} & \leq V_{s}+M_{p, T}\left|x_{1}-x_{2}\right|^{1 / 2}\left(\int_{\mathbb{R}} G_{t-s}^{2}(u) d u\right)^{1 / 2} \\
& =V_{s}+M_{p, T} \sqrt{\delta}[8(t-s)]^{-1 / 4} .
\end{aligned}
$$

Substituting the estimate (3.9) into (3.8) yields

$$
\begin{aligned}
\left\|I_{2,+}\left(x_{1}, x_{2}\right)\right\|_{p}^{2} & \leq 2 c_{p}^{2}\|f\|_{L^{2}(\mathbb{R})}^{2} \int_{0}^{t}\left(V_{s}^{2}+8^{-1 / 2} M_{p, T}^{2} \delta(t-s)^{-1 / 2}\right) d s \\
& \leq 2 c_{p}^{2}\|f\|_{L^{2}(\mathbb{R})}^{2}\left(\int_{0}^{t} V_{s}^{2} d s+\sqrt{\frac{T}{2}} M_{p, T}^{2} \delta\right) .
\end{aligned}
$$

An analogous upper bound can be obtained for $\left\|I_{2,-}\left(x_{1}, x_{2}\right)\right\|_{p}^{2}$ in the same way. Similarly, decompose $I_{3}$ as

$$
I_{3}\left(x_{1}, x_{2}\right)=I_{3,+}\left(x_{1}, x_{2}\right)+I_{3,-}\left(x_{1}, x_{2}\right),
$$

where

$$
I_{3,+}\left(x_{1}, x_{2}\right)=\int_{0}^{t} \int_{0}^{\infty} \sigma_{s}^{2}(y) \int_{y}^{\infty}\left[G_{t-s}\left(x_{1}-z\right)-G_{t-s}\left(x_{2}-z\right)\right] \psi(s, z) d z d y d s
$$

and

$$
I_{3,-}\left(x_{1}, x_{2}\right)=\int_{0}^{t} \int_{-\infty}^{0} \sigma_{s}^{2}(y) \int_{-\infty}^{y}\left[G_{t-s}\left(x_{1}-z\right)-G_{t-s}\left(x_{2}-z\right)\right] \psi(s, z) d z d y d s .
$$

By Minkowsky inequality,

$$
\left\|I_{3,+}\left(x_{1}, x_{2}\right)\right\|_{p} \leq \int_{0}^{t} \int_{0}^{\infty} f^{2}(y)\left\|\int_{y}^{\infty}\left[G_{t-s}\left(x_{1}-z\right)-G_{t-s}\left(x_{2}-z\right)\right] \psi(s, z) d z\right\|_{p} d y d s .
$$


and the estimate (3.9) leads to

$$
\left\|I_{3,+}\left(x_{1}, x_{2}\right)\right\|_{p} \leq\|f\|_{L^{2}(\mathbb{R})}^{2}\left(\int_{0}^{t} V_{s} d s+\frac{4}{3} T^{3 / 4} M_{p, T} 8^{-1 / 4} \sqrt{\delta}\right) .
$$

We can derive an analogous estimate for $\left\|I_{3,-}\left(x_{1}, x_{2}\right)\right\|_{p}$. Finally, from (3.7), (3.10), (3.11), and the similar estimates for $I_{2,-}$ and $I_{3,-}$, we deduce

$$
V_{t}^{2} \leq C_{1} \delta+C_{2} \int_{0}^{t} V_{s}^{2} d s
$$

for some constants $C_{1}$ and $C_{2}$ depending on $p, T,\left\|u_{0}\right\|_{\infty},\left\|u_{0}\right\|_{L^{1}(\mathbb{R})}$ and $\|f\|_{L^{2}(\mathbb{R})}$. By Gronwall's lemma, $V_{t} \leq C \sqrt{\delta}$, which implies the desired Hölder continuity in the space variable.

For time regularity, let $0 \leq t_{1}<t_{2} \leq T$ and consider each of the decomposition

$$
\psi\left(t_{2}, x\right)-\psi\left(t_{1}, x\right)=J_{1}\left(t_{1}, t_{2}\right)-\frac{1}{2} J_{2}\left(t_{1}, t_{2}\right)+\frac{1}{8} J_{3}\left(t_{1}, t_{2}\right),
$$

where

$$
\begin{gathered}
J_{1}\left(t_{1}, t_{2}\right)=\int_{\mathbb{R}}\left(G_{t_{2}}(x-y)-G_{t_{1}}(x-y)\right) \psi_{0}(y) d y \\
J_{2}\left(t_{1}, t_{2}\right)=\int_{0}^{t_{2}} \int_{S} \operatorname{sign}(y) G_{t_{2}-s}(x-z) \psi(s, z) \sigma_{s}(y) d z W(d s, d y) \\
-\int_{0}^{t_{1}} \int_{S} \operatorname{sign}(y) G_{t_{1}-s}(x-z) \psi(s, z) \sigma_{s}(y) d z W(d s, d y),
\end{gathered}
$$

and

$$
\begin{aligned}
J_{3}\left(t_{1}, t_{2}\right)= & \int_{0}^{t_{2}} \int_{S}\left[G_{t_{2}-s}(x-z)-G_{t-s}(x-z)\right] \psi(s, z) \sigma_{s}^{2}(y) d z d y d s \\
& -\int_{0}^{t_{1}} \int_{S}\left[G_{t_{1}-s}(x-z)-G_{t-s}(x-z)\right] \psi(s, z) \sigma_{s}^{2}(y) d z d y d s .
\end{aligned}
$$

Apply the semigroup property and the Lipschitz property of $\psi_{0}$ to get

$$
\begin{aligned}
\left|J_{1}\left(t_{1}, t_{2}\right)\right| & =\left|\int_{\mathbb{R}} G_{t_{1}}(x-y)\left(\int_{\mathbb{R}} G_{t_{2}-t_{1}}(y-z)\left[\psi_{0}(z)-\psi_{0}(y)\right] d z\right) d y\right| \\
& \leq C \int_{\mathbb{R}} G_{t_{1}}(x-y)\left(\int_{\mathbb{R}} G_{t_{2}-t_{1}}(y-z)|z-y| d z\right) d y \\
& =C\left(t_{2}-t_{1}\right)^{1 / 2} .
\end{aligned}
$$

For the stochastic integral term, we again decompose $J_{2}$ as

$$
J_{2}\left(t_{1}, t_{2}\right)=J_{2,+}\left(t_{1}, t_{2}\right)-J_{2,-}\left(t_{1}, t_{2}\right)
$$

where

$$
\begin{aligned}
J_{2,+}\left(t_{1}, t_{2}\right)= & \int_{0}^{t_{2}} \int_{0}^{\infty} \int_{y}^{\infty} G_{t_{2}-s}(x-z) \psi(s, z) \sigma_{s}(y) W(d s, d y) \\
& -\int_{0}^{t_{1}} \int_{0}^{\infty} \int_{y}^{\infty} G_{t_{1}-s}(x-z) \psi(s, z) \sigma_{s}(y) W(d s, d y)
\end{aligned}
$$


and

$$
\begin{aligned}
J_{2,-}\left(t_{1}, t_{2}\right)= & \int_{0}^{t_{2}} \int_{-\infty}^{0} \int_{-\infty}^{y} G_{t_{2}-s}(x-z) \psi(s, z) \sigma_{s}(y) W(d s, d y) \\
& -\int_{0}^{t_{1}} \int_{-\infty}^{0} \int_{-\infty}^{y} G_{t_{1}-s}(x-z) \psi(s, z) \sigma_{s}(y) W(d s, d y) .
\end{aligned}
$$

Splitting $J_{2,+}$ into two pieces, we can write

$$
\begin{aligned}
& \left\|J_{2,+}\left(t_{1}, t_{2}\right)\right\|_{p} \\
& \leq\left\|\int_{0}^{t_{1}} \int_{0}^{\infty} \sigma_{s}(y)\left(\int_{y}^{\infty} \psi(s, z)\left[G_{t_{2}-s}(x-z)-G_{t_{1}-s}(x-z)\right] d z\right) W(d s, d y)\right\|_{p} \\
& \quad+\left\|\int_{t_{1}}^{t_{2}} \int_{0}^{\infty} \sigma_{s}(y)\left(\int_{y}^{\infty} \psi(s, z) G_{t_{2}-s}(x-z) d z\right) W(d s, d y)\right\|_{p} \\
& =: A_{1}\left(t_{1}, t_{2}\right)+A_{2}\left(t_{1}, t_{2}\right) .
\end{aligned}
$$

Applying Burkholder's inequality and Minkowski's inequality, yields

$$
A_{1}\left(t_{1}, t_{2}\right) \leq c_{p}\left(\int_{0}^{t_{1}} \int_{0}^{\infty} f(y)^{2}\left\|\int_{y}^{\infty} \psi(s, z)\left[G_{t_{2}-s}(x-z)-G_{t_{1}-s}(x-z)\right] d z\right\|_{p}^{2} d y d s\right)^{1 / 2} .
$$

Adding and subtracting $\psi(s, x)$ and using the spatial regularity of $\psi$, we obtain

$$
\begin{aligned}
& \left\|\int_{y}^{\infty} \psi(s, z)\left[G_{t_{2}-s}(x-z)-G_{t_{1}-s}(x-z)\right] d z\right\|_{p}^{2} \\
& \quad \leq 2 C\left(\int_{y}^{\infty}\left|G_{t_{2}-s}(x-z)-G_{t_{1}-s}(x-z)\right||x-z|^{1 / 2} d z\right)^{2} \\
& \quad+2\|\psi(s, x)\|_{p}^{2}\left(\int_{y}^{\infty}\left[G_{t_{2}-s}(x-z)-G_{t_{1}-s}(x-z)\right] d z\right)^{2} .
\end{aligned}
$$

By Lemma 2.1, with $\beta=2, \theta_{1}=1$ and $\theta_{2}=1 / 2$, yields

$$
\int_{0}^{t_{1}}\left(\int_{y}^{\infty}\left|G_{t_{2}-s}(x-z)-G_{t_{1}-s}(x-z)\right||x-z|^{1 / 2} d z\right)^{2} d s \leq C\left(t_{2}-t_{2}\right)^{3 / 2} .
$$

Applying Lemma 2.1 again, with $\beta=2, \theta_{1}=1$ and $\theta_{2}=0$, we obtain

$$
\int_{0}^{t_{1}}\left(\int_{y}^{\infty}\left[G_{t_{2}-s}(x-z)-G_{t_{1}-s}(x-z)\right] d z\right)^{2} d s \leq C\left(t_{2}-t_{1}\right) .
$$

Substituting (3.13) and (3.14) into (3.12), we get

$$
A_{1}\left(t_{1}, t_{2}\right) \leq C\left(t_{2}-t_{1}\right)^{1 / 2} .
$$


We control the term $A_{2}\left(t_{1}, t_{2}\right)$ using a rough estimate as follows

$$
\begin{aligned}
A_{2}\left(t_{1}, t_{2}\right) & \leq c_{p}\left\|\int_{t_{1}}^{t_{2}} \int_{0}^{\infty} f(y)^{2}\left(\int_{y}^{\infty} \psi(s, z) G_{t_{2}-s}(x-z) d z\right)^{2} d y d s\right\|_{p / 2}^{1 / 2} \\
& \leq c_{p}\|f\|_{L^{2}(\mathbb{R})}\left(\int_{t_{1}}^{t_{2}}\left\|\int_{\mathbb{R}} \psi(s, z) G_{t_{2}-s}(x-z) d z\right\|_{p}^{2} d s\right)^{1 / 2} \\
& \leq C\left(t_{2}-t_{1}\right)^{1 / 2} .
\end{aligned}
$$

We can bound $J_{2,-}$ in the same way and get

$$
\left\|J_{2}\left(t_{1}, t_{2}\right)\right\|_{p} \leq C\left(t_{2}-t_{1}\right)^{1 / 2}
$$

Once again, we decompose $J_{3}$ as $J_{3}=J_{3,+}+J_{3,-}$, where

$$
\begin{aligned}
J_{3,+}\left(t_{1}, t_{2}\right)= & \int_{0}^{t_{2}} \int_{0}^{\infty} \int_{y}^{\infty} G_{t_{2}-s}(s, z) \psi(s, z) \sigma_{s}^{2}(y) d z d y d s \\
& -\int_{0}^{t_{1}} \int_{0}^{\infty} \int_{y}^{\infty} G_{t_{1}-s}(s, z) \psi(s, z) \sigma_{s}^{2}(y) d z d y d s
\end{aligned}
$$

and

$$
\begin{aligned}
J_{3,-}\left(t_{1}, t_{2}\right)=\int_{0}^{t_{2}} & \int_{-\infty}^{0} \int_{-\infty}^{y} G_{t_{2}-s}(s, z) \psi(s, z) \sigma_{s}^{2}(y) d z d y d s \\
& -\int_{0}^{t_{1}} \int_{-\infty}^{0} \int_{-\infty}^{y} G_{t_{1}-s}(s, z) \psi(s, z) \sigma_{s}^{2}(y) d z d y d s .
\end{aligned}
$$

We control $J_{3,+}$ in the same way as $J_{2,+}$ to get

$$
\begin{gathered}
\left\|J_{3,+}\left(t_{1}, t_{2}\right)\right\|_{p} \leq\left\|\int_{0}^{t_{1}} \int_{0}^{\infty} \sigma_{s}(y)^{2} \int_{y}^{\infty} \psi(s, z)\left[G_{t_{2}-s}(x-z)-G_{t_{1}-s}(x-z)\right] d z d y d s\right\|_{p} \\
+\left\|\int_{t_{1}}^{t_{2}} \int_{0}^{\infty} \sigma_{s}(y)^{2} \int_{y}^{\infty} \psi(s, z) G_{t_{2}-s}(x-z) d z d y d s\right\|_{p}
\end{gathered}
$$

We bound the second term roughly as

$$
\begin{aligned}
\left\|\int_{t_{1}}^{t_{2}} \int_{0}^{\infty} \sigma_{s}(y)^{2} \int_{y}^{\infty} \psi(s, z) G_{t_{2}-s}(x-z) d z d y d s\right\|_{p} & \leq C \int_{t_{1}}^{t_{2}} \int_{\mathbb{R}} G_{t_{2}-s}(x-z) d z d s \\
& =C\left(t_{2}-t_{1}\right) .
\end{aligned}
$$

Then, notice that for any $\epsilon \in(0,1)$ the first term can be bounded as

$$
\begin{aligned}
\int_{0}^{t_{1}} & \int_{\mathbb{R}}\left|G_{t_{2}-s}(x-z)-G_{t_{1}-s}(x-z)\right| d z d s \\
\leq & C \int_{0}^{t_{1}}\left(\int_{\mathbb{R}}\left|G_{t_{2}-s}(x)-G_{t_{1}-s}(x)\right|^{p_{1}(1-\epsilon)} d x\right)^{1 / p_{1}} \\
& \times\left[\left(\int_{\mathbb{R}} G_{t_{2}-s}(x)^{p_{2} \epsilon} d x\right)^{1 / p_{2}}+\left(\int_{\mathbb{R}} G_{t_{1}-s}(x)^{p_{2} \epsilon} d x\right)^{1 / p_{2}}\right] d s \\
\leq & C \int_{0}^{t_{1}}\left(\int_{\mathbb{R}}\left|G_{t_{2}-s}(x)-G_{t_{1}-s}(x)\right|^{p_{1}(1-\epsilon)} d x\right)^{1 / p_{1}},
\end{aligned}
$$


for any Hölder conjugates $p_{1}, p_{2}$. Notice that if $\beta=1 / p_{1}, \theta_{1}=p_{1}(1-\epsilon)$, and $\theta_{2}=0$, then condition (2.3) is satisfied when, for example, $\epsilon=1 / p_{1}$ and $p_{1}>4$. Hence, using Lemma 2.1 with these parameters yields

$$
\int_{0}^{t_{1}} \int_{\mathbb{R}}\left|G_{t_{2}-s}(x-z)-G_{t_{1}-s}(x-z)\right| d z d s \leq C\left(t_{2}-t_{1}\right)^{1 / 2+1 / p_{1}} .
$$

Control $J_{3,-}$ in an identical way to obtain

$$
\left\|J_{3}\left(t_{1}, t_{2}\right)\right\|_{p} \leq C\left(t_{2}-t_{1}\right)^{1 / 2}
$$

Combining the above estimates yields

$$
\left\|\psi\left(t_{2}, x\right)-\psi\left(t_{1}, x\right)\right\|_{p} \leq C\left(t_{2}-t_{1}\right)^{1 / 2} .
$$

Next we use the established Hölder regularity of the process $\psi$ to study its spatial differentiability.

Proposition 3.5. The process $\psi(t, \cdot)$ is differentiable in $L^{p}(\Omega)$ for any $p \geq 2$ and satisfies

$$
\begin{array}{r}
\frac{\partial \psi}{\partial x}(t, x)=\int_{\mathbb{R}} \frac{\partial G_{t}}{\partial x}(x-y) \psi_{0}(y) d y-\frac{1}{2} \int_{0}^{t} \int_{S} \operatorname{sign}(y) \frac{\partial G_{t-s}}{\partial x}(x-z) \psi(s, z) \sigma_{s}(y) d z W(d s, d y) \\
+\frac{1}{8} \int_{0}^{t} \int_{S} \frac{\partial G_{t-s}}{\partial x}(x-z) \psi(s, z) \sigma_{s}(y)^{2} d z d y d s .
\end{array}
$$

Proof. It is clear that the spatial derivative of the first integral in the expression of $\psi$ equals the first integral above by Leibniz's rule.

To take care of the stochastic integral term, by the Burkholder-Davis-Gundy inequality and the symmetry of $S$, it suffices to show the convergence to zero in $L^{p / 2}(\Omega)$, as $h$ tends to zero, of the term

$$
I_{h}(t, x):=\int_{0}^{t} \int_{0}^{\infty}\left(\int_{y}^{\infty} \Delta_{h} G_{t-s}(x-z) \psi(s, z) d z\right)^{2} \sigma_{s}(y)^{2} d y d s,
$$

where

$$
\Delta_{h} G_{t-s}(x-z):=\frac{G_{t-s}(x+h-z)-G_{t-s}(x-z)}{h}-\frac{\partial G_{t-s}}{\partial x}(x-z) .
$$

By Minkowski's inequality, we obtain

$$
\left\|I_{h}(t, x)\right\|_{p / 2} \leq \int_{0}^{t} \int_{0}^{\infty}\left\|\int_{y}^{\infty} \Delta_{h} G_{t-s}(x-z) \psi(s, z) d z\right\|_{p}^{2} f(y)^{2} d y d s .
$$

We show first the convergence to zero of

$$
I_{h}(t, x, s):=\int_{0}^{\infty}\left\|\int_{y}^{\infty} \Delta_{h} G_{t-s}(x-z) \psi(s, z) d z\right\|_{p}^{2} f(y)^{2} d y
$$

as $h$ tends to zero, for each fixed $s \in[0, t)$. Rough estimates of $I_{h}(t, x, s)$ lead to

$$
I_{h}(t, x, s) \leq \sup _{t, x}\|\psi(t, x)\|_{p}^{2}\|f\|_{L^{2}(\mathbb{R})}^{2}\left(\int_{\mathbb{R}}\left|\Delta_{h} G_{t-s}(z)\right| d z\right)^{2} .
$$


Apply the mean value theorem twice to see that

$$
\Delta_{h} G_{t-s}(z)=\frac{1}{h} \int_{0}^{h} \int_{0}^{u} \frac{\partial^{2} G_{t-s}}{\partial x^{2}}(z+\eta) d \eta d u .
$$

Finally, by applying Fubini's theorem, we obtain

$$
I_{h}(t, x, s) \leq C_{s}|h|^{2} .
$$

Hence, we have that, for each $s \in[0, t), I_{h}(t, x, s) \rightarrow 0$ as $h \rightarrow 0$. By the dominated convergence theorem, it now suffices to show that $I_{h}(t, x, s)$ is bounded by a $d s$-integrable function which is independent of $h$. Again, by the mean value theorem, we can write

$$
I_{h}(t, x, s)=\int_{0}^{\infty}\left\|\int_{y}^{\infty} \frac{1}{h} \int_{0}^{h}\left(\frac{\partial G_{t-s}}{\partial x}(x+\xi-z)-\frac{\partial G_{t-s}}{\partial x}(x-z)\right) d \xi \psi(s, z) d z\right\|_{p}^{2} f(y)^{2} d y .
$$

We split up this quantity by adding and subtracting appropriate terms as follows

$$
I_{h}(t, x, s)=\int_{0}^{\infty}\left\|\int_{y}^{\infty}\left[\phi_{1}(s, x, z, h)+\phi_{2}(s, x, z, h)\right] d z\right\|_{p}^{2} f(y)^{2} d y,
$$

where

$$
\begin{aligned}
\phi_{1}(s, x, z, h):= & \frac{1}{h} \int_{0}^{h} \frac{\partial G_{t-s}}{\partial x}(x+\xi-z)[\psi(s, z)-\psi(s, x+\xi)] d \xi \\
& -\frac{\partial G_{t-s}}{\partial x}(x-z)[\psi(s, z)-\psi(s, x)]
\end{aligned}
$$

and

$$
\phi_{2}(s, x, z, h):=\frac{1}{h} \int_{0}^{h}\left[\frac{\partial G_{t-s}}{\partial x}(x+\xi-z) \psi(s, x+\xi)-\frac{\partial G_{t-s}}{\partial x}(x-z) \psi(s, x)\right] d \xi .
$$

Let us first consider the two terms of $\phi_{1}$, one at a time. For the first one, we can write, using Minkowski inequality and the Hölder continuity in $L^{p}(\Omega)$ of $\psi$

$$
\begin{aligned}
\int_{0}^{\infty} \| & \int_{y}^{\infty} \frac{1}{h} \int_{0}^{h} \frac{\partial G_{t-s}}{\partial x}(x+\xi-z)[\psi(s, z)-\psi(s, x+\xi)] d \xi d z \|_{p}^{2} f(y)^{2} d y \\
& \leq C\|f\|_{L^{2}(\mathbb{R})}^{2} \frac{1}{h^{2}}\left(\int_{0}^{h} \int_{\mathbb{R}}\left|\frac{\partial G_{t-s}}{\partial x}(x+\xi-z)\right||x+\xi-z|^{1 / 2} d z d \xi\right)^{2} \\
& =C\|f\|_{L^{2}(\mathbb{R})}^{2}(t-s)^{-1 / 2}
\end{aligned}
$$

which is $d s$-integrable. Now, to see that the second term is also bounded by a $d s$-integrable function not depending on $h$, we bound in the same way to get

$$
\begin{aligned}
\int_{0}^{\infty} \| & \int_{y}^{\infty} \frac{\partial G_{t-s}}{\partial x}(x-z)[\psi(s, z)-\psi(s, x)] d z \|_{p}^{2} f(y)^{2} d y \\
& \leq C \int_{0}^{\infty}\left(\int_{y}^{\infty}\left|\frac{\partial G_{t-s}}{\partial x}(x-z)\right||z-x|^{1 / 2} d z\right)^{2} f(y)^{2} d y \\
& \leq C\|f\|_{L^{2}(\mathbb{R})}^{2}(t-s)^{-1 / 2} .
\end{aligned}
$$


Let us now control the term $\phi_{2}$ by first interchanging the $d \xi$ and $d z$ integrals to get

$$
\begin{aligned}
\int_{0}^{\infty} & \left\|\int_{y}^{\infty} \phi_{2}(s, x, z, h) d z\right\|_{p}^{2} f(y)^{2} d y \\
& =\int_{0}^{\infty}\left\|\frac{1}{h} \int_{0}^{h}\left[G_{t-s}(x+\xi-y) \psi(s, x+\xi)-G_{t-s}(x-y) \psi(s, x)\right] d \xi\right\|_{p}^{2} f(y)^{2} d y .
\end{aligned}
$$

Now, add and subtract $G_{t-s}(x+\xi-y) \psi(s, x)$ to get

$$
\begin{aligned}
\int_{0}^{\infty}\left\|\int_{y}^{\infty} \phi_{2}(s, x, z, h) d z\right\|_{p}^{2} f(y)^{2} d y \\
\leq 2 \int_{0}^{\infty}\left\|\psi(s, x) \frac{1}{h} \int_{0}^{h}\left[G_{t-s}(x+\xi-y)-G_{t-s}(x-y)\right] d \xi\right\|_{p}^{2} f(y)^{2} d y \\
\quad \quad+2 \int_{0}^{\infty}\left\|\frac{1}{h} \int_{0}^{h} G_{t-s}(x+\xi-y)[\psi(s, x+\xi)-\psi(s, x)] d \xi\right\|_{p}^{2} f(y)^{2} d y \\
=: J_{1, h}+J_{2, h} .
\end{aligned}
$$

The second term can easily be bounded as follows

$$
J_{2, h} \leq\left.\left. C \int_{\mathbb{R}} f(y)^{2}\left|\frac{1}{h} \int_{0}^{h} G_{t-s}(x+\xi-y)\right| \xi\right|^{1 / 2} d \xi\right|^{2} d y
$$

We now use the assumption $f \in L^{q}(\mathbb{R})$ for some $q>2$ and choose $p_{1}$ such that $\frac{1}{p_{1}}+\frac{2}{q}=1$. Then, by Hölder's inequality, we can write

$$
J_{2, h} \leq C\|f\|_{L^{q}(\mathbb{R})}^{2}\left\|\frac{1}{h} \int_{0}^{h} G_{t-s}(x+\xi-\cdot)|\xi|^{1 / 2} d \xi\right\|_{L^{2 p_{1}(\mathbb{R})}}^{2} .
$$

Now, by Minkowski's inequality, we have

$$
\left\|\frac{1}{h} \int_{0}^{h} G_{t-s}(x+\xi-\cdot)|\xi|^{1 / 2} d \xi\right\|_{L^{2 p_{1}(\mathbb{R})}}^{2} \leq C(t-s)^{-1+1 / 2 p_{1}}\left(\frac{1}{h} \int_{0}^{h}|\xi|^{1 / 2} d \xi\right)^{2},
$$

which is $d s$-integrable and independent of $h$ since we can assume $|h| \leq 1$ without loss of generality. Finally, to control $J_{1, h}$, we proceed by again choosing the same value of $p_{1}$ :

$$
\begin{aligned}
J_{1, h} & \leq C\|f\|_{L^{q(\mathbb{R})}}^{2}\left(\frac{1}{h} \int_{0}^{h}\left\|G_{t-s}(x+\xi-\cdot)-G_{t-s}(x, \cdot)\right\|_{L^{2 p_{1}(\mathbb{R})}} d \xi\right)^{2} \\
& \leq C\|f\|_{L^{q(\mathbb{R})}}^{2}(t-s)^{-1+1 /\left(2 p_{1}\right)}
\end{aligned}
$$

which is $d s$-integrable.

For the third integral in the expression of $\partial_{x} \psi$, we use an identical argument to obtain pointwise convergence to zero. Furthermore, it is easy to bound the $d s$ integrand by an integrable function which is independent of $h$ since

$$
\begin{aligned}
& \left\|\int_{0}^{\infty} \int_{y}^{\infty} \Delta_{h} G_{t-s}(x-z) \psi(s, z) \sigma_{s}(y)^{2} d z d y\right\|_{p} \leq C\|f\|_{L^{2}(\mathbb{R})}^{2} \int_{\mathbb{R}}\left|\Delta_{h} G_{t-s}(x-z)\right| d z \\
& \quad \leq C \int_{\mathbb{R}}\left(\left|\frac{\partial G_{t-s}}{\partial x}(x+\xi-z)\right|+\left|\frac{\partial G_{t-s}}{\partial x}(x-z)\right|\right) d z \\
& \quad=C(t-s)^{-1 / 2}
\end{aligned}
$$


where the second inequality follows from the mean value theorem and triangle inequality.

In order to obtain a continuity result for the derivative process given above, we first establish uniform moment bounds.

Proposition 3.6. For all integers $p \geq 2$, we have for any $t \geq 0$,

$$
\sup _{x \in \mathbb{R}}\left\|\frac{\partial \psi}{\partial x}(t, x)\right\|_{p} \leq K c_{p}(t \vee 1)^{1-1 / q} \exp \left(c_{p}\|f\|_{L^{2}(\mathbb{R})}^{2} t+\frac{1}{2}\|f\|_{L^{2}(\mathbb{R})}^{4} t^{2}\right),
$$

where $c_{p}$ is the optimal constant in Burkholder's inequality and $K$ is a constant depending on $p, q,\|f\|_{L^{q}(\mathbb{R})},\|f\|_{L^{2}(\mathbb{R})},\left\|u_{0}\right\|_{L^{1}(\mathbb{R})}$ and $\left\|u_{0}\right\|_{\infty}$.

Proof. From the integral equation (3.15) satisfied by $\frac{\partial \psi}{\partial x}(t, x)$, we get the decomposition

$$
\frac{\partial \psi}{\partial x}(t, x)=\mathcal{I}_{1}(t, x)-\mathcal{I}_{2}(t, x)+\mathcal{I}_{3}(t, x),
$$

where

$$
\begin{gathered}
\mathcal{I}_{1}(t, x)=\int_{\mathbb{R}} \frac{\partial G_{t}}{\partial x}(x-y) \psi_{0}(y) d y \\
\mathcal{I}_{2}(t, x)=\frac{1}{2} \int_{0}^{t} \int_{S} \operatorname{sign}(y) \frac{\partial G_{t-s}}{\partial x}(x-z) \psi(s, z) \sigma_{s}(y) d z W(d s, d y)
\end{gathered}
$$

and

$$
\mathcal{I}_{3}(t, x)=\frac{1}{8} \int_{0}^{t} \int_{S} \frac{\partial G_{t-s}}{\partial x}(x-z) \psi(s, z) \sigma_{s}(y)^{2} d z d y d s .
$$

First observe that integrating by parts yields

$$
\left|\mathcal{I}_{1}(t, x)\right| \leq \int_{\mathbb{R}} G_{t}(x-y)\left|\frac{\partial \psi_{0}}{\partial y}(y)\right| d y \leq \frac{1}{2}\left\|u_{0}\right\|_{\infty} e^{\frac{1}{2}\left\|u_{0}\right\|_{L^{1}(\mathbb{R})}} .
$$

Now, decompose $\mathcal{I}_{2}$ as $\mathcal{I}_{2}(t, x)=\mathcal{I}_{2,+}(t, x)+\mathcal{I}_{2,-}(t, x)$, where

$$
\mathcal{I}_{2,+}(t, x)=\int_{0}^{t} \int_{0}^{\infty} \int_{y}^{\infty} \frac{\partial G_{t-s}}{\partial x}(x-z) \psi(s, z) \sigma_{s}(y) d z W(d s, d y)
$$

and

$$
\mathcal{I}_{2,-}(t, x)=-\int_{0}^{t} \int_{-\infty}^{0} \int_{-\infty}^{y} \frac{\partial G_{t-s}}{\partial x}(x-z) \psi(s, z) \sigma_{s}(y) d z W(d s, d y) .
$$

Using Burkholder's and Minkowski's inequalities, we get

$$
\left\|\mathcal{I}_{2,+}(t, x)\right\|_{p}^{2} \leq c_{p} \int_{0}^{t} \int_{0}^{\infty}\left\|\int_{y}^{\infty} \frac{\partial G_{t-s}}{\partial x}(x-z) \psi(s, z) d z\right\|_{p}^{2} f(y)^{2} d y d s .
$$

Integrate by parts, use the triangle inequality, and the uniform bounds on moments of $\psi$ to obtain

$$
\begin{aligned}
\left\|\int_{y}^{\infty} \frac{\partial G_{t-s}}{\partial x}(x-z) \psi(s, z) d z\right\|_{p}^{2} & \leq 2 M_{p, t}^{2} G_{t-s}^{2}(x-y) \\
& +2\left(\int_{\mathbb{R}} G_{t-s}(x-z)\left\|\frac{\partial \psi}{\partial z}(s, z)\right\|_{p} d z\right)^{2},
\end{aligned}
$$


where $M_{p, t}=\sup _{x \in \mathbb{R}}\|\psi(t, x)\|_{p}$. By Hölder's inequality, if $\frac{1}{q_{1}}+\frac{2}{q}=1$, then

$$
\begin{aligned}
\int_{\mathbb{R}} f(y)^{2} G_{t-s}^{2}(x-y) d y & \leq\left(\int_{\mathbb{R}} G_{t-s}(x-y)^{2 q_{1}} d y\right)^{1 / q_{1}}\|f\|_{L^{q}(\mathbb{R})}^{2} \\
& =k\|f\|_{L^{q(\mathbb{R})}}^{2}(t-s)^{-1+1 /\left(2 q_{1}\right)},
\end{aligned}
$$

where $k$ is a universal constant. Let

$$
U_{t}:=\sup _{x \in \mathbb{R}}\left\|\frac{\partial \psi}{\partial x}(t, x)\right\|_{p}^{2} .
$$

The above estimates yield

$$
\begin{aligned}
\left\|\mathcal{I}_{2,+}(t, x)\right\|_{p}^{2} & \leq \frac{4 q}{q-2} c_{p} k\|f\|_{L^{q}(\mathbb{R})}^{2} M_{p, t}^{2} t^{\frac{1}{2}-\frac{1}{q}}+2 c_{p}\|f\|_{L^{2}(\mathbb{R})}^{2} \int_{0}^{t} U_{s} d s \\
& =c_{p, t}^{(1)}+c_{p}^{(2)} \int_{0}^{t} U_{s} d s
\end{aligned}
$$

where $c_{p, t}^{(1)}$ and $c_{p}^{(2)}$ are positive constants depending on $p, q, t,\|f\|_{L^{q}(\mathbb{R})},\|f\|_{L^{2}(\mathbb{R})}$ and $\left\|u_{0}\right\|_{L^{1}(\mathbb{R})}$. We obtain the same bound on $\left\|\mathcal{I}_{2,-}(t, x)\right\|_{p}$ in an identical way. Similarly, $\mathcal{I}_{3}(t, x)=\mathcal{I}_{3,+}(t, x)+\mathcal{I}_{3,-}(t, x)$ where

$$
\mathcal{I}_{3,+}(t, x)=\int_{0}^{t} \int_{0}^{\infty} \int_{y}^{\infty} \frac{\partial G_{t-s}}{\partial x}(x-z) \psi(s, z) \sigma_{s}(y)^{2} d z d y d s
$$

and

$$
\mathcal{I}_{3,-}(t, x)=\int_{0}^{t} \int_{-\infty}^{0} \int_{-\infty}^{y} \frac{\partial G_{t-s}}{\partial x}(x-z) \psi(s, z) \sigma_{s}(y)^{2} d z d y d s .
$$

Again, integrating by parts, using Minkowski's inequality, and Proposition 3.1, we obtain

$$
\begin{aligned}
\left\|\mathcal{I}_{3,+}(t, x)\right\|_{p} & \leq \int_{0}^{t} \int_{0}^{\infty} f(y)^{2}\left(M_{p, t} G_{t-s}(x-y)+\int_{y}^{\infty} G_{t-s}(x-z)\left\|\frac{\partial \psi}{\partial z}(s, z)\right\|_{p} d z\right) d y d s \\
& \leq M_{p, t}\|f\|_{L^{q}(\mathbb{R})}^{2} \int_{0}^{t}\left\|G_{t-s}\right\|_{L^{q_{1}(\mathbb{R})}} d s+\|f\|_{L^{2}(\mathbb{R})}^{2} \int_{0}^{t} \sup _{x \in \mathbb{R}}\left\|\frac{\partial \psi}{\partial x}(s, x)\right\|_{p} d s .
\end{aligned}
$$

Hence, we have

$$
\begin{aligned}
\left\|\mathcal{I}_{3,+}(t, x)\right\|_{p}^{2} & \leq k M_{p, t}^{2}\|f\|_{L^{q}(\mathbb{R})}^{4}\left(\frac{q}{q-1}\right)^{2} t^{2-2 / q}+2 t\|f\|_{L^{2}(\mathbb{R})}^{4} \int_{0}^{t} U_{s} d s \\
& =c_{p, t}^{(3)}+c_{p, t}^{(4)} \int_{0}^{t} U_{s} d s
\end{aligned}
$$

for some constants $c_{p, t}^{(3)}$ and $c_{p, t}^{(4)}$. We can bound $\mathcal{I}_{3,-}$ in the same way. Putting each bound from above together and applying Gronwall's inequality, we obtain the desired result.

Proposition 3.7. Suppose that in addition to condition (A1), the initial condition $u_{0}$ is Hölder continuous of order $\alpha \in[0,1]$. Then, for any $p \geq 2$ and any $T>0$, there exists some constant $C$, depending on $p, T, u_{0}$, and $f$, such that for all $s, t \in[0, T]$, and $x, y \in \mathbb{R}$,

$$
\left\|\frac{\partial \psi}{\partial x}(t, x)-\frac{\partial \psi}{\partial y}(s, y)\right\|_{p} \leq C\left(|t-s|^{\frac{\alpha}{2} \wedge\left(\frac{1}{4}-\frac{1}{2 q}\right)}+|x-y|^{\alpha \wedge\left(\frac{1}{2}-\frac{1}{q}\right)}\right),
$$


where $q$ is the exponent appearing in Assumption (A2).

Proof. We first study Hölder continuity in the space variable. Fix $t \in[0, T]$, let $x_{1}, x_{2} \in \mathbb{R}$ be given, and set $\delta=\left|x_{1}-x_{2}\right|$. Without loss of generality we can assume that $\delta \leq 1$. We consider spatial increments of each term in (3.16) one at a time. The first term is easily controlled integrating by parts and using the fact that $\psi_{0}^{\prime}$ is Hölder continuous of order $\alpha$ :

$$
\left|\mathcal{I}_{1}\left(t, x_{1}\right)-\mathcal{I}_{1}\left(t, x_{2}\right)\right| \leq \int_{\mathbb{R}} G_{t}(y)\left|\psi_{0}^{\prime}\left(x_{1}-y\right)-\psi_{0}^{\prime}\left(x_{2}-y\right)\right| d y \leq C \delta^{\alpha} .
$$

For the second term, we again use the decomposition $\mathcal{I}_{2}(t, x)=\mathcal{I}_{2,+}(t, x)+\mathcal{I}_{2,-}(t, x)$, where $\mathcal{I}_{2,+}$ and $\mathcal{I}_{2,-}$ have been introduced in (3.17) and (3.18), respectively. Integrating by parts, we obtain

$$
\begin{aligned}
\int_{y}^{\infty} & {\left[\frac{\partial G_{t-s}}{\partial x}\left(x_{1}-z\right)-\frac{\partial G_{t-s}}{\partial x}\left(x_{2}-z\right)\right] \psi(s, z) d z } \\
= & {\left[G_{t-s}\left(x_{1}-y\right)-G_{t-s}\left(x_{2}-y\right)\right] \psi(s, y) } \\
& +\int_{y}^{\infty}\left[G_{t-s}\left(x_{1}-z\right)-G_{t-s}\left(x_{2}-z\right)\right] \frac{\partial \psi}{\partial z}(s, z) d z \\
= & : \mathcal{I}_{2,+}^{A}\left(t-s, x_{1}, x_{2}, y\right)+\mathcal{I}_{2,+}^{B}\left(t-s, x_{1}, x_{2}, y\right) .
\end{aligned}
$$

Applying Burkholder's inequality, (2.2), Minkowski's inequality, and Proposition 3.1, we get

$$
\begin{aligned}
& \left\|\int_{0}^{t} \int_{0}^{\infty} \mathcal{I}_{2,+}^{A}\left(t-s, x_{1}, x_{2}, y\right) \sigma_{s}(y) W(d s, d y)\right\|_{p}^{2} \\
& \quad \leq C \int_{0}^{t} \int_{\mathbb{R}}\left|G_{t-s}\left(x_{1}-y\right)-G_{t-s}\left(x_{2}-y\right)\right|^{2} f(y)^{2} d y d s \\
& \quad \leq C\|f\|_{L^{q}(\mathbb{R})}^{2} \int_{0}^{t}\left(\int_{\mathbb{R}}\left|G_{t-s}\left(x_{1}-y\right)-G_{t-s}\left(x_{2}-y\right)\right|^{2 q_{1}} d y\right)^{1 / q_{1}} d s,
\end{aligned}
$$

where $\frac{2}{q}+\frac{1}{q_{1}}=1$. Making the substitutions $y=\delta z$ and $t-s=\delta^{2} v$, yields

$$
\begin{aligned}
\int_{0}^{t} \| & {\left[G_{t-s}\left(x_{1}-\cdot\right)-G_{t-s}\left(x_{2}-\cdot\right)\right]^{2} \|_{L^{q_{1}(\mathbb{R})}} d s } \\
\leq & \leq \delta^{1 / q_{1}} \int_{0}^{\infty} v^{-1}\left(\int_{\mathbb{R}}\left|\exp \left(-(1+z)^{2} / 4 v\right)-\exp \left(-z^{2} / 4 v\right)\right|^{2 q_{1}} d z\right)^{1 / q_{1}} d v \\
& =C \delta^{1 / q_{1}} .
\end{aligned}
$$

Therefore, from (3.21) and (3.22), we obtain

$$
\left\|\int_{0}^{t} \int_{0}^{\infty} \mathcal{I}_{2,+}^{A}\left(t-s, x_{1}, x_{2}, y\right) \sigma_{s}(y) W(d s, d y)\right\|_{p}^{2} \leq C\|f\|_{L^{q(\mathbb{R})}}^{2} \delta^{1 / q_{1}} .
$$

To handle $\mathcal{I}_{2,+}^{B}$, we use the same techniques as in the proof of Proposition 3.4 to first write

$$
\begin{gathered}
\mathcal{I}_{2,+}^{B}\left(t-s, x_{1}, x_{2}, y\right)=\int_{-\infty}^{x_{1}-y} G_{t-s}(u)\left[\frac{\partial \psi}{\partial z}\left(s, x_{1}-u\right)-\frac{\partial \psi}{\partial z}\left(s, x_{2}-u\right)\right] d u \\
+\int_{x_{2}-y}^{x_{1}-y} \frac{\partial \psi}{\partial z}\left(s, x_{2}-u\right) G_{t-s}(u) d u
\end{gathered}
$$


Let

$$
\widetilde{V}_{s}:=\sup _{|x-y|=\delta}\left\|\frac{\partial \psi}{\partial x}(s, x)-\frac{\partial \psi}{\partial x}(s, y)\right\|_{p}
$$

and

$$
N_{p}:=\sup _{t, x}\left\|\frac{\partial \psi}{\partial x}(t, x)\right\|_{p}
$$

Then, we can write

$$
\begin{aligned}
\left\|\mathcal{I}_{2,+}^{B}\left(t-s, x_{1}, x_{2}, y\right)\right\|_{p} & \leq \widetilde{V}_{s}+N_{p} \int_{x_{2}-y}^{x_{1}-y} G_{t-s}(u) d u \\
& \leq \widetilde{V}_{s}+N_{p} \sqrt{\delta}[8(t-s)]^{-1 / 4}
\end{aligned}
$$

Hence,

$$
\left\|\int_{0}^{t} \int_{0}^{\infty} \mathcal{I}_{2,+}^{B}\left(t-s, x_{1}, x_{2}, y\right) \sigma_{s}(y) W(d s, d y)\right\|_{p}^{2} \leq C\|f\|_{L^{2}(\mathbb{R})}^{2}\left(\int_{0}^{t} \widetilde{V}_{s}^{2} d s+\delta N_{p}^{2}\right) .
$$

Therefore, from (3.23) and (3.24), we get

$$
\left\|\mathcal{I}_{2,+}\left(t, x_{1}\right)-\mathcal{I}_{2,+}\left(t, x_{2}\right)\right\|_{p}^{2} \leq C\|f\|_{L^{q}(\mathbb{R})}^{2} \delta^{1 / q_{1}}+C\|f\|_{L^{2}(\mathbb{R})}^{2}\left(\delta N_{p}^{2}+\int_{0}^{t} \widetilde{V}_{s}^{2} d s\right) .
$$

We can get the same bounds on increments of $\mathcal{I}_{2,-}$ in an identical way. Once again, write $\mathcal{I}_{3}=\mathcal{I}_{3,+}+\mathcal{I}_{3,-}$, as in (3.19) and (3.20). Integrate by parts, and use the same techniques as above to get

$$
\begin{aligned}
\left\|\mathcal{I}_{3,+}\left(t, x_{1}\right)-\mathcal{I}_{3,+}\left(t, x_{2}\right)\right\|_{p} \leq & C\left(\int_{0}^{t} \int_{\mathbb{R}}\left|G_{t-s}\left(x_{1}-y\right)-G_{t-s}\left(x_{2}-y\right)\right| f(y)^{2} d y d s\right. \\
& \left.+C\|f\|_{L^{2}(\mathbb{R})}^{2} \sqrt{\delta} N_{p}+\|f\|_{L^{2}(\mathbb{R})}^{2} \int_{0}^{t} \widetilde{V}_{s} d s\right) \\
\leq & C\left(\|f\|_{L^{q}(\mathbb{R})}^{2}+\|f\|_{L^{2}(\mathbb{R})}^{2} \sqrt{\delta} N_{p}+\|f\|_{L^{2}(\mathbb{R})}^{2} \int_{0}^{t} \widetilde{V_{s}} d s\right) .
\end{aligned}
$$

The same bounds for increments of $\mathcal{I}_{3,-}$ are obtained the same way. Put all of these pieces together by taking the smallest power of $\delta$ to get

$$
\tilde{V}_{t}^{2} \leq C\left(\delta^{2 \alpha}+\delta^{1-2 / q}+\int_{0}^{t} \widetilde{V}_{s}^{2} d s\right)
$$

Thus, Gronwall's inequality implies that $x \mapsto \frac{\partial \psi}{\partial x}(t, x)$ is Hölder continuous in $L^{p}(\Omega)$, uniformly in $t$, with order of regularity $\alpha \wedge(1 / 2-1 / q)$.

To establish regularity in time, fix $0 \leq t_{1}<t_{2} \leq T$ and write

$$
\begin{aligned}
\left|\mathcal{I}_{1}\left(t_{2}, x\right)-\mathcal{I}_{1}\left(t_{1}, x\right)\right| & =\left|\int_{\mathbb{R}} G_{t_{1}}(x-y)\left(\int_{\mathbb{R}} G_{t_{2}-t_{1}}(y-z)\left[\psi_{0}^{\prime}(z)-\psi_{0}^{\prime}(y)\right] d z\right) d y\right| \\
& \leq C \int_{\mathbb{R}} G_{t_{1}}(x-y)\left(\int_{\mathbb{R}} G_{t_{2}-t_{1}}(y-z)|y-z|^{\alpha} d z\right) d y \\
& =C\left(t_{2}-t_{1}\right)^{\alpha / 2} .
\end{aligned}
$$


Then, we again split up $\left\|\mathcal{I}_{2,+}\left(t_{2}, x\right)-\mathcal{I}_{2,+}\left(t_{1}, x\right)\right\|_{p}$ into two terms as

$$
\begin{aligned}
& \left\|\mathcal{I}_{2,+}\left(t_{2}, x\right)-\mathcal{I}_{2,+}\left(t_{1}, x\right)\right\|_{p} \\
& \leq\left\|\int_{0}^{t_{1}} \int_{0}^{\infty} \sigma_{s}(y)\left(\int_{y}^{\infty} \psi(s, z) \frac{\partial}{\partial x}\left[G_{t_{2}-s}(x-z)-G_{t_{1}-s}(x-z)\right] d z\right) W(d s, d y)\right\|_{p} \\
& \quad+\left\|\int_{t_{1}}^{t_{2}} \int_{0}^{\infty} \sigma_{s}(y)\left(\int_{y}^{\infty} \psi(s, z) \frac{\partial}{\partial x} G_{t_{2}-s}(x-z) d z\right) W(d s, d y)\right\|_{p} \\
& =: \widetilde{J}_{1}+\widetilde{J}_{2} .
\end{aligned}
$$

Integrate by parts, and apply Burkholder's and Minkowski's inequalities to get

$$
\begin{aligned}
\widetilde{J}_{2} \leq & c_{p}\left(\int_{t_{1}}^{t_{2}} \int_{0}^{\infty}\left\|G_{t_{2}-s}(x-y) \psi(s, y)+\int_{y}^{\infty} G_{t_{2}-s}(x-z) \frac{\partial \psi}{\partial z}(s, z) d z\right\|_{p}^{2} f(y)^{2} d y d s\right)^{1 / 2} \\
\leq C & \left(\int_{t_{1}}^{t_{2}} \int_{\mathbb{R}} G_{t_{2}-s}(x-y)^{2} f(y)^{2} d y d s\right. \\
& \left.\quad+\int_{t_{1}}^{t_{2}} \int_{0}^{\infty} f(y)^{2}\left\|\int_{y}^{\infty} G_{t_{2}-s}(x-z) \frac{\partial \psi}{\partial z}(s, z) d z\right\|_{p}^{2} d y d s\right)^{1 / 2} .
\end{aligned}
$$

By Hölder's inequality, we have

$$
\int_{t_{1}}^{t_{2}} \int_{\mathbb{R}} G_{t_{2}-s}(x-y)^{2} f(y)^{2} d y d s \leq \int_{t_{1}}^{t_{2}}\left\|G_{t_{2}-s}\right\|_{L^{2 q_{1}(\mathbb{R})}}^{2}\|f\|_{L^{q}(\mathbb{R})}^{2} d s=C\left(t_{2}-t_{1}\right)^{1 /\left(2 q_{1}\right)}
$$

where $\frac{1}{q_{1}}+\frac{2}{q}=1$. For the other term, we make use of the uniform bounds on moments of the derivative of $\psi$ to get

$$
\int_{t_{1}}^{t_{2}} \int_{0}^{\infty} f(y)^{2}\left\|\int_{y}^{\infty} G_{t_{2}-s}(x-z) \frac{\partial \psi}{\partial z}(s, z) d z\right\|_{p}^{2} d y d s \leq C\left(t_{2}-t_{1}\right)
$$

for some constant $C$. Hence,

$$
\widetilde{J}_{2} \leq C\left|t_{2}-t_{1}\right|^{1 /\left(4 q_{1}\right)}
$$

For the term $\widetilde{J}_{1}$, we first apply Burkholder's inequality and integrate by parts to get

$$
\begin{aligned}
\widetilde{J}_{1} \leq & C\left(\int_{0}^{t_{1}} \int_{0}^{\infty} f(y)^{2}\|\psi(s, y)\|_{p}^{2}\left[G_{t_{2}-s}(x-y)-G_{t_{1}-s}(x-y)\right]^{2} d y d s\right. \\
& \left.+\int_{0}^{t_{1}} \int_{0}^{\infty}\left\|\int_{y}^{\infty}\left[G_{t_{2}-s}(x-z)-G_{t_{1}-s}(x-z)\right] \frac{\partial \psi}{\partial z}(s, z) d z\right\|_{p}^{2} f(y)^{2} d y d s\right)^{1 / 2} \\
= & C\left(\widetilde{J}_{1,1}+\widetilde{J}_{1,2}\right)^{\frac{1}{2}} .
\end{aligned}
$$

Using the uniform bounds on $\psi$, choosing $q_{1}$ such that $\frac{1}{q_{1}}+\frac{2}{q}=1$, and applying Lemma 2.1 with $\beta=1 / q_{1}, \theta_{1}=2 q_{1}$ and $\theta_{2}=0$, we can write

$$
\begin{aligned}
\widetilde{J}_{1,1} & \leq C\|f\|_{L^{q}(\mathbb{R})}^{2} \int_{0}^{t_{1}}\left(\int_{\mathbb{R}}\left|G_{t_{2}-s}(y)-G_{t_{1}-s}(y)\right|^{2 q_{1}} d y\right)^{\frac{1}{q_{1}}} d s \\
& \leq C\|f\|_{L^{q(\mathbb{R})}}^{2}\left(t_{2}-t_{1}\right)^{1 /\left(2 q_{1}\right)} .
\end{aligned}
$$


For the term $\widetilde{J}_{1,2}$, we same techniques as in the proof of the Hölder regularity in time of $\psi$ by first adding and subtracting $\frac{\partial \psi}{\partial x}(s, x)$ and applying the spatial regularity of the derivative of $\psi$ to get

$$
\begin{aligned}
\widetilde{J}_{1,2} \leq & 2\|f\|_{L^{2}(\mathbb{R})}^{2} \int_{0}^{t_{1}}\left(\int_{\mathbb{R}}\left|G_{t_{2}-s}(x-z)-G_{t_{1}-s}(x-z) \| x-z\right|^{\alpha \wedge\left(\frac{1}{2}-\frac{1}{q}\right)} d z\right)^{2} d s \\
& +2 \int_{0}^{t_{1}} \int_{0}^{\infty}\left\|\frac{\partial \psi}{\partial x}(s, x)\right\|_{p}^{2}\left(\int_{y}^{\infty}\left[G_{t_{2}-s}(x-z)-G_{t_{1}-s}(x-z)\right] d z\right)^{2} f(y)^{2} d y d s .
\end{aligned}
$$

We apply Lemma 2.1 with $\beta=2, \theta_{1}=1$, and $\theta_{2}=\alpha \wedge\left(\frac{1}{2}-\frac{1}{q}\right)$ to get

$$
\int_{0}^{t_{1}}\left(\int_{\mathbb{R}}\left|G_{t_{2}-s}(x-z)-G_{t_{1}-s}(x-z)\right||x-z|^{\alpha \wedge\left(\frac{1}{2}-\frac{1}{q}\right)} d z\right)^{2} d s \leq C\left(t_{2}-t_{1}\right)^{1+\alpha \wedge\left(\frac{1}{2}-\frac{1}{q}\right)} .
$$

Another application of Lemma 2.1 with $\beta=2, \theta_{1}=1$ and $\theta_{2}=0$, yields

$$
\int_{0}^{t_{1}}\left(\int_{y}^{\infty}\left[G_{t_{2}-s}(x-z)-G_{t_{1}-s}(x-z)\right] d z\right)^{2} d s \leq C\left(t_{2}-t_{1}\right) .
$$

Hence,

$$
\widetilde{J}_{1} \leq C\left(t_{2}-t_{1}\right)^{1 /\left(4 q_{1}\right)}
$$

Put these together to get

$$
\left\|\mathcal{I}_{2,+}\left(t_{2}, x\right)-\mathcal{I}_{2,+}\left(t_{1}, x\right)\right\|_{p} \leq C\left(t_{2}-t_{1}\right)^{1 / 4-1 /(2 q)} .
$$

We can obtain the same upper bound for $I_{2,-}$ and hence

$$
\left\|\mathcal{I}_{2}\left(t_{2}, x\right)-\mathcal{I}_{2}\left(t_{1}, x\right)\right\|_{p} \leq C\left(t_{2}-t_{1}\right)^{1 / 4-1 /(2 q)} .
$$

For the third term, we apply the same techniques we used for $\mathcal{I}_{2}$ to get

$$
\left\|\mathcal{I}_{3}\left(t_{2}, x\right)-\mathcal{I}_{3}\left(t_{1}, x\right)\right\|_{p} \leq C\left(t_{2}-t_{1}\right)^{1 / 4} .
$$

Hence, we have the desired result.

Remark 3.8. If we do not assume the Hölder continuity of $u_{0}$, then $\psi_{0}^{\prime}$ is only continuous. Then, avoiding the integration by parts in the proof of the Hölder continuity of the first term, we have a result of the form

$$
\left\|\frac{\partial \psi}{\partial x}(t, x)-\frac{\partial \psi}{\partial y}(s, y)\right\|_{p} \leq C(t \wedge s)^{-1 / 2}\left(|t-s|^{\frac{1}{4}-\frac{1}{2 q}}+|x-y|^{\frac{1}{2}-\frac{1}{q}}\right)
$$

where the factor $t^{-1 / 2}$, assuming $t \leq s$, comes from the integral $\int_{\mathbb{R}}\left|\frac{\partial G_{t}}{\partial t}(y)\right| d y$. That is, the Hölder continuity blows up at $t=0$. However, $\frac{\partial \psi}{\partial x}(t, x)$ is continuous in $L^{p}(\Omega)$ on $\mathbb{R}_{+} \times \mathbb{R}$ for all $p \geq 2$, because $\psi_{0}^{\prime}$ is continuous. 


\section{Hopf-Cole Transformation}

In this section, we construct a solution to Burgers' equation (1.1) using the Hopf-Cole transformation and the results of the previous section. Notice first that the process

$$
v(t, x):=-2 \frac{\partial}{\partial x} \log \psi(t, x)=-\frac{2}{\psi(t, x)} \frac{\partial \psi}{\partial x}(t, x)
$$

is well defined and has uniformly bounded moments of order $p$ for all $p \geq 2$, due to Proposition 3.6 and Remark 3.2. We now establish the main result of the paper which asserts that the process $v(t, x)$ is the solution to the Burgers' equation (1.1). Again, uniqueness follows for free from [3].

The main idea of the proof is to introduce the regularized process

$$
\psi_{\epsilon}(t, x):=\int_{\mathbb{R}} G_{\epsilon}(x-y) \psi(t, y) d y,
$$

for $\epsilon \in(0,1]$ and to find the equation satisfied by $u_{\epsilon}(t, x):=-2 \frac{\partial}{\partial x} \log \psi_{\epsilon}(t, x)$. Based on previous results, it is easy to see that $\psi_{\epsilon}$ satisfies the following property.

Lemma 4.1. For any $p \geq 2$ and $T>0$, we have

$$
\sup _{x \in \mathbb{R}, \epsilon \in(0,1], t \in[0, T]}\left(\left\|\psi_{\epsilon}(t, x)\right\|_{p}+\left\|\psi_{\epsilon}(t, x)^{-1}\right\|_{p}+\left\|\frac{\partial \psi_{\epsilon}}{\partial x}(t, x)\right\|_{p}\right)<\infty .
$$

For any $p \geq 2, x \in \mathbb{R}$, and $t \in(0, T]$, we have

$$
\left\|\psi(t, x)-\psi_{\epsilon}(t, x)\right\|_{p} \leq C \epsilon^{1 / 4}
$$

and

$$
\left\|\frac{\partial \psi}{\partial x}(t, x)-\frac{\partial \psi_{\epsilon}}{\partial x}(t, x)\right\|_{p} \leq C t^{-1 / 2} \epsilon^{1 / 4-1 /(2 q)} .
$$

Proof. Inequality (4.1) follows form Jensen's inequality, Propositions 3.1 and 3.6, and Remark 3.2. Inequalities (4.2) and (4.3) are consequences of Proposition 3.4 and Remark 3.8.

Theorem 4.2. The process $v(t, x)=-2 \frac{\partial}{\partial x} \log \psi(t, x)$ is a solution to (1).

Proof. From Proposition 3.3, we have that $\psi_{\epsilon}$ satisfies

$$
\begin{aligned}
\psi_{\epsilon}(t, x)= & \int_{\mathbb{R}} G_{t+\epsilon}(x-y) \psi_{0}(y) d y-\frac{1}{2} \int_{0}^{t} \int_{S} \operatorname{sign}(y) G_{t+\epsilon-s}(x-z) \psi(s, z) \sigma_{s}(y) d z W(d s, d y) \\
& +\frac{1}{8} \int_{0}^{t} \int_{S} G_{t+\epsilon-s}(x-z) \psi(s, z) \sigma_{s}(y)^{2} d z d y d s .
\end{aligned}
$$

Next, apply the semigroup property of the heat kernel to get

$$
\begin{aligned}
\psi_{\epsilon}(t, x)= & \int_{\mathbb{R}} G_{t}(x-z)\left(\int_{\mathbb{R}} G_{\epsilon}(z-y) \psi_{0}(y) d y\right) d z \\
& -\frac{1}{2} \int_{0}^{t} \int_{S} \int_{\mathbb{R}} \operatorname{sign}(v) G_{t-s}(x-y) G_{\epsilon}(y-z) \psi(s, z) \sigma_{s}(v) d y d z W(d s, d v) \\
& +\frac{1}{8} \int_{0}^{t} \int_{S} \int_{\mathbb{R}} G_{t-s}(x-y) G_{\epsilon}(y-z) \psi(s, z) \sigma_{s}(v)^{2} d y d z d v d s .
\end{aligned}
$$


Note that this is the mild formulation of the following stochastic heat equation

$$
\begin{aligned}
\psi_{\epsilon}(t, x)= & \int_{\mathbb{R}} G_{\epsilon}(x-y) \psi_{0}(y) d y+\int_{0}^{t} \frac{\partial^{2} \psi_{\epsilon}}{\partial x^{2}}(s, x) d s \\
& -\frac{1}{2} \int_{0}^{t} \int_{S} \operatorname{sign}(y) G_{\epsilon}(x-z) \psi(s, z) \sigma_{s}(y) d z W(d s, d y) \\
& +\frac{1}{8} \int_{0}^{t} \int_{S} G_{\epsilon}(x-z) \psi(s, z) \sigma_{s}(y)^{2} d z d y d s .
\end{aligned}
$$

The process $t \rightarrow \psi_{\epsilon}(t, x)$ is a semimartingale and applying Itô's formula to $\log \psi_{\epsilon}(t, x)$ yields

$$
\begin{aligned}
\log \psi_{\epsilon}(t, x)= & \log \left(\int_{\mathbb{R}} G_{\epsilon}(x-y) \psi_{0}(y) d y\right)+\int_{0}^{t} \frac{1}{\psi_{\epsilon}(s, x)} \frac{\partial^{2} \psi_{\epsilon}}{\partial x^{2}}(s, x) d s \\
& -\frac{1}{2} \int_{0}^{t} \int_{S} \operatorname{sign}(y) \frac{1}{\psi_{\epsilon}(s, x)} G_{\epsilon}(x-z) \psi(s, z) \sigma_{s}(y) d z W(d s, d y) \\
& +\frac{1}{8} \int_{0}^{t} \int_{S} \frac{1}{\psi_{\epsilon}(s, x)} G_{\epsilon}(x-z) \psi(s, z) \sigma_{s}(y)^{2} d z d y d s \\
- & \frac{1}{8} \int_{0}^{t} \int_{\mathbb{R}} \frac{1}{\psi_{\epsilon}(s, x)^{2}} \Psi_{\epsilon}(s, x, y)^{2} \sigma_{s}(y)^{2} d y d s,
\end{aligned}
$$

where

$$
\Psi_{\epsilon}(s, x, y):=\mathbf{1}_{(y \geq 0)} \int_{y}^{\infty} G_{\epsilon}(x-z) \psi(s, z) d z+\mathbf{1}_{(y<0)} \int_{-\infty}^{y} G_{\epsilon}(x-z) \psi(s, z) d z .
$$

Now, noting that basic calculus gives $\frac{1}{f} \frac{\partial^{2} f}{\partial x^{2}}=\frac{\partial^{2}}{\partial x^{2}}(\log f)+\left(\frac{\partial}{\partial x} \log f\right)^{2}$, we have

$$
\begin{aligned}
\frac{\partial}{\partial x} \log \psi_{\epsilon}(t, x)= & \frac{\partial}{\partial x} \log \left(\int_{\mathbb{R}} G_{\epsilon}(x-y) \psi_{0}(y) d y\right) \\
+ & \int_{0}^{t} \frac{\partial^{2}}{\partial x^{2}}\left(\frac{\partial}{\partial x} \log \psi_{\epsilon}(s, x)\right) d s+\int_{0}^{t} \frac{\partial}{\partial x}\left(\left(\frac{\partial}{\partial x} \log \psi_{\epsilon}(s, x)\right)^{2}\right) d s \\
& -\frac{1}{2} \int_{0}^{t} \int_{S} \operatorname{sign}(y) \frac{\partial}{\partial x}\left(\frac{1}{\psi_{\epsilon}(s, x)} G_{\epsilon}(x-z)\right) \psi(s, z) \sigma_{s}(y) d z W(d s, d y) \\
& +\frac{1}{8} \int_{0}^{t} \int_{S} \frac{\partial}{\partial x}\left(\frac{1}{\psi_{\epsilon}(s, x)} G_{\epsilon}(x-z)\right) \psi(s, z) \sigma_{s}(y)^{2} d z d y d s \\
& -\frac{1}{8} \int_{0}^{t} \int_{\mathbb{R}} \frac{\partial}{\partial x}\left(\frac{1}{\psi_{\epsilon}(s, x)} \Psi_{\epsilon}(s, x, y)\right)^{2} \sigma_{s}(y)^{2} d y d s .
\end{aligned}
$$


So, the process $u_{\epsilon}(t, x):=-2 \frac{\partial}{\partial x} \log \psi_{\epsilon}(t, x)$ satisfies the following integral equation

$$
\begin{aligned}
u_{\epsilon}(t, x) & =\int_{\mathbb{R}} G_{t}(x-y) u_{\epsilon}(0, y) d y-\frac{1}{2} \int_{0}^{t} \int_{\mathbb{R}} G_{t-s}(x-y) \frac{\partial}{\partial y} u_{\epsilon}(s, y)^{2} d y d s \\
& +\int_{0}^{t} \int_{S} \int_{\mathbb{R}} \operatorname{sign}(y) G_{t-s}(x-v) \frac{\partial}{\partial v}\left(\frac{1}{\psi_{\epsilon}(s, v)} G_{\epsilon}(v-z)\right) \psi(s, z) \sigma_{s}(y) d v d z W(d s, d y) \\
& -\frac{1}{4} \int_{0}^{t} \int_{S} \int_{\mathbb{R}} G_{t-s}(x-v) \frac{\partial}{\partial v}\left(\frac{1}{\psi_{\epsilon}(s, v)} G_{\epsilon}(v-z)\right) \psi(s, z) \sigma_{s}(y)^{2} d v d y d z d s \\
& +\frac{1}{4} \int_{0}^{t} \int_{\mathbb{R}} \int_{\mathbb{R}} G_{t-s}(x-v) \frac{\partial}{\partial v}\left(\frac{1}{\psi_{\epsilon}(s, v)} \Psi_{\epsilon}(s, v, y)\right)^{2} \sigma_{s}(y)^{2} d v d y d s .
\end{aligned}
$$

Finally, integration by parts yields

$$
\begin{aligned}
u_{\epsilon}(t, x)= & \int_{\mathbb{R}} G_{t}(x-y) u_{\epsilon}(0, y) d y+\frac{1}{2} \int_{0}^{t} \int_{\mathbb{R}} \frac{\partial}{\partial y} G_{t-s}(x-y) u_{\epsilon}(s, y)^{2} d y d s \\
& -\int_{0}^{t} \int_{S} \int_{\mathbb{R}} \operatorname{sign}(y) \frac{\partial}{\partial v} G_{t-s}(x-v) \frac{1}{\psi_{\epsilon}(s, v)} G_{\epsilon}(v-z) \psi(s, z) \sigma_{s}(y) d v d z W(d s, d y) \\
& +\frac{1}{4} \int_{0}^{t} \int_{S} \int_{\mathbb{R}} \frac{\partial}{\partial v} G_{t-s}(x-v) \frac{1}{\psi_{\epsilon}(s, v)} G_{\epsilon}(v-z) \psi(s, z) \sigma_{s}(y)^{2} d v d z d y d s \\
& -\frac{1}{4} \int_{0}^{t} \int_{\mathbb{R}} \int_{\mathbb{R}} \frac{\partial}{\partial v} G_{t-s}(x-v) \frac{1}{\psi_{\epsilon}^{2}(s, v)} \Psi_{\epsilon}(x, v, y)^{2} \sigma_{s}(y)^{2} d v d y d s \\
= & \sum_{i=1}^{5} A_{i, \epsilon} .
\end{aligned}
$$

We will study the convergence of each term in the above expression. This will be done in several steps:

Step 1. For the term $A_{1, \epsilon}$, taking into account that

$$
u_{\epsilon}(0, x)=-2 \frac{\left(\psi_{0}^{\prime} * G_{\epsilon}\right)(x)}{\left(\psi_{0} * G_{\epsilon}\right)(x)}
$$

and $\psi_{0}^{\prime}$ is continuous and bounded, it is easy to show that

$$
A_{1, \epsilon} \rightarrow \int_{\mathbb{R}} G_{t}(x-y) u_{0}(y) d y
$$

as $\epsilon$ tends to zero.

Step 2. From Lemma 4.1 it follows that

$$
\left\|u_{\epsilon}(t, x)-v(t, x)\right\|_{p} \leq C t^{-1 / 2} \epsilon^{\frac{1}{4}-\frac{1}{2 q}} .
$$

With this, it is easy to see that

$$
A_{2, \epsilon} \rightarrow \frac{1}{2} \int_{0}^{t} \int_{\mathbb{R}} \frac{\partial}{\partial y} G_{t-s}(x-y) v(s, y)^{2} d y d s,
$$

as $\epsilon \rightarrow 0$, in $L^{p}(\Omega)$ for all $p \geq 2$. 
Step 3. We now show the convergence of the stochastic integral term $A_{3, \epsilon}$. Integrating by parts, first with respect to $v$, then with respect to $z$, we get for $y>0$,

$$
\begin{gathered}
\int_{\mathbb{R}} \frac{\partial}{\partial v} G_{t-s}(x-v) \frac{1}{\psi_{\epsilon}(s, v)}\left(\int_{y}^{\infty} G_{\epsilon}(v-z) \psi(s, z) d z\right) d v \\
=\int_{\mathbb{R}} G_{t-s}(x-v) \frac{1}{\psi_{\epsilon}(s, v)^{2}} \frac{\partial \psi_{\epsilon}}{\partial v}(s, v)\left(\int_{y}^{\infty} G_{\epsilon}(v-z) \psi(s-z) d z\right) d v \\
\quad-\int_{\mathbb{R}} G_{t-s}(x-v) \frac{1}{\psi_{\epsilon}(s, v)}\left(\int_{y}^{\infty} \frac{\partial G_{\epsilon}}{\partial v}(v-z) \psi(s, z) d z\right) d v \\
=\int_{\mathbb{R}} G_{t-s}(x-v) \frac{1}{\psi_{\epsilon}(s, v)^{2}} \frac{\partial \psi_{\epsilon}}{\partial v}(s, v)\left(\int_{y}^{\infty} G_{\epsilon}(v-z) \psi(s, z) d z\right) d v \\
-\int_{\mathbb{R}} G_{t-s}(x-v) \frac{1}{\psi_{\epsilon}(s, v)}\left(\int_{y}^{\infty} G_{\epsilon}(v-z) \frac{\partial \psi}{\partial z}(s, z) d z\right) d v \\
-\psi(s, y) \int_{\mathbb{R}} G_{t-s}(x-v) \frac{1}{\psi_{\epsilon}(s, v)} G_{\epsilon}(v-y) d v \\
=: G_{1,+, \epsilon}(s, y)-G_{2,+, \epsilon}(s, y)-G_{3, \epsilon}(y, s) .
\end{gathered}
$$

In a similar way, for $y<0$, we obtain

$$
\begin{aligned}
\int_{\mathbb{R}} \frac{\partial}{\partial v} G_{t-s}(x-v) & \frac{1}{\psi_{\epsilon}(s, v)}\left(\int_{-\infty}^{y} G_{\epsilon}(v-z) \psi(s, z) d z\right) d v \\
& =G_{1,-, \epsilon}(s, y)-G_{2,-, \epsilon}(s, y)+G_{3, \epsilon}(y, s)
\end{aligned}
$$

where the terms $G_{1,-, \epsilon}(s, y)$ and $G_{2,-, \epsilon}(s, y)$ are analogous to $G_{1,+, \epsilon}(s, y)$ and $G_{2,+, \epsilon}(s, y)$, respectively, by just replacing the integral $\int_{y}^{\infty}$ by $\int_{-\infty}^{y}$.

We claim that the following convergences hold in $L^{p}(\Omega)$, for any $p \geq 2$, as $\epsilon \rightarrow 0$ :

$$
\begin{gathered}
\int_{0}^{t} \int_{\mathbb{R}} G_{3, \epsilon}(s, y) \sigma_{s}(y) W(d s, d y) \rightarrow \int_{0}^{t} \int_{\mathbb{R}} G_{t-s}(x-y) \sigma_{s}(y) W(d s, d y), \\
\int_{0}^{t} \int_{\mathbb{R}}\left[G_{1,+, \epsilon}(s, y)-G_{2,+, \epsilon}(s, y)\right] \sigma_{s}(y) W(d s, d y) \rightarrow 0 .
\end{gathered}
$$

and

$$
\int_{0}^{t} \int_{\mathbb{R}}\left[G_{1,-, \epsilon}(s, y)-G_{2,-, \epsilon}(s, y)\right] \sigma_{s}(y) W(d s, d y) \rightarrow 0 .
$$

Proof of (4.4): Applying Burkholder's inequality and Minkowski's inequality, we can write

$$
\begin{aligned}
\| \int_{0}^{t} \int_{\mathbb{R}^{2}} & G_{\epsilon}(v-y)\left(\frac{\psi(s, y)}{\psi_{\epsilon}(s, v)} G_{t-s}(x-v)-G_{t-s}(x-y)\right) \sigma_{s}(y) d v W(d s, d y) \|_{p}^{2} \\
& \leq C \int_{0}^{t} \int_{\mathbb{R}}\left\|\int_{\mathbb{R}} G_{\epsilon}(v-y) \frac{\psi(s, y)}{\psi_{\epsilon}(s, v)} G_{t-s}(x-v) d v-G_{t-s}(x-y)\right\|_{p}^{2} f(y)^{2} d y d s \\
& \leq C\left(B_{1, \epsilon}+B_{2, \epsilon}\right),
\end{aligned}
$$


where

$$
B_{1, \epsilon}=\int_{0}^{t} \int_{\mathbb{R}}\left(\int_{\mathbb{R}} G_{\epsilon}(v-y) G_{t-s}(x-v)\left\|\frac{\psi(s, y)}{\psi_{\epsilon}(s, v)}-1\right\|_{p} d v\right)^{2} f(y)^{2} d y d s
$$

and

$$
B_{2, \epsilon}=\int_{0}^{t} \int_{\mathbb{R}}\left(G_{t-s+\epsilon}(x-y)-G_{t-s}(x-y)\right)^{2} f(y)^{2} d y d s .
$$

Using the definition of $\psi_{\epsilon}$ and 3.4, it is not difficult to see that $\psi_{\epsilon}$ is Hölder continuous of order $1 / 2$ in the spatial variable. With this and Lemma 4.1, we have

$$
\left\|\frac{\psi(s, y)}{\psi_{\epsilon}(s, v)}-1\right\|_{p} \leq C\left(\epsilon^{1 / 4}+|y-v|^{1 / 2}\right) .
$$

Therefore,

$$
\begin{aligned}
B_{1, \epsilon} \leq C \epsilon^{1 / 2} & \int_{0}^{t} \int_{\mathbb{R}} G_{t+\epsilon-s}^{2}(x-y) f(y)^{2} d y d s \\
& +C \int_{0}^{t} \int_{\mathbb{R}}\left(\int_{\mathbb{R}} G_{\epsilon}(v-y) G_{t-s}(x-v)|v-y|^{1 / 2} d v\right)^{2} f(y)^{2} d y d s .
\end{aligned}
$$

Clearly,

$$
\int_{0}^{t} \int_{\mathbb{R}} G_{t+\epsilon-s}^{2}(x-y) f(y)^{2} d y d s \leq C,
$$

by Hölder's inequality and assumption (A.2). Next, make the change of variables $v-y=z$ and choose $q_{1}>1$ such that $\frac{1}{q_{1}}+\frac{2}{q}=1$, to get

$$
\begin{aligned}
\int_{0}^{t} \int_{\mathbb{R}} & \left(\int_{\mathbb{R}} G_{\epsilon}(v-y) G_{t-s}(x-v)|v-y|^{1 / 2} d v\right)^{2} f(y)^{2} d y d s \\
& \leq\|f\|_{L^{q}(\mathbb{R})}^{2} \int_{0}^{t}\left(\int_{\mathbb{R}} G_{\epsilon}(z)|z|^{1 / 2} d z\right)^{2}\left\|G_{t-s}\right\|_{L^{2 q_{1}(\mathbb{R})}}^{2} d s \\
& \leq C \epsilon^{1 / 2} .
\end{aligned}
$$

Hence, $B_{1, \epsilon} \rightarrow 0$ as $\epsilon \rightarrow 0$. On the other hand, again using Lemma 2.1, yields

$$
B_{2, \epsilon} \leq C\|f\|_{L^{q}(\mathbb{R})}^{2} \epsilon^{1 / 2-1 / q} .
$$

Proof of (4.5): Adding and subtracting $\psi(s, v)$ and $\frac{\partial \psi}{\partial v}(s, v)$ in the $d z$ integrals of the first and second terms, respectively, we get the decomposition

$$
\int_{0}^{t} \int_{\mathbb{R}}\left[G_{1,+, \epsilon}(s, y)-G_{2,+, \epsilon}(s, y)\right] \sigma_{s}(y) W(d s, d y)=J_{1, \epsilon}+J_{2, \epsilon}+J_{3, \epsilon},
$$

where

$$
\begin{aligned}
J_{1, \epsilon} & =\int_{0}^{t} \int_{0}^{\infty} \int_{\mathbb{R}} \frac{G_{t-s}(x-v)}{\psi_{\epsilon}^{2}(s, v)} \frac{\partial \psi_{\epsilon}}{\partial v}(s, v)\left(\int_{y}^{\infty} G_{\epsilon}(v-z)[\psi(s, z)-\psi(s, v)] d z\right) d v \sigma_{s}(y) W(d s, d y), \\
J_{2, \epsilon} & =\int_{0}^{t} \int_{0}^{\infty} \int_{\mathbb{R}} \frac{G_{t-s}(x-v)}{\psi_{\epsilon}(s, v)}\left(\int_{y}^{\infty} G_{\epsilon}(v-z)\left[\frac{\partial \psi}{\partial z}(s, z)-\frac{\partial \psi}{\partial v}(s, v)\right] d z\right) d v \sigma_{s}(y) W(d s, d y)
\end{aligned}
$$


and

$$
\begin{array}{r}
J_{3, \epsilon}=\int_{0}^{t} \int_{0}^{\infty} \int_{\mathbb{R}} \frac{G_{t-s}(x-v)}{\psi_{\epsilon}^{2}(s, v)}\left[\psi(s, v) \frac{\partial \psi_{\epsilon}}{\partial v}(s, v)-\psi_{\epsilon}(s, v) \frac{\partial \psi}{\partial v}(s, v)\right] \\
\times\left(\int_{y}^{\infty} G_{\epsilon}(v-z) d z\right) d v \sigma_{s}(y) W(d s, d y) .
\end{array}
$$

Applying Burkholder and Minkowski inequalities yields, for any $p \geq 2$,

$$
\begin{aligned}
\left\|J_{1, \epsilon}\right\|_{p}^{2} \leq & c_{p} \int_{0}^{t} \int_{0}^{\infty}\left(\int_{\mathbb{R}} G_{t-s}(x-v)\left\|\frac{1}{\psi_{\epsilon}^{2}(s, v)} \frac{\partial \psi_{\epsilon}}{\partial v}(s, v)\right\|_{2 p}\right. \\
& \left.\times \int_{\mathbb{R}} G_{\epsilon}(v-z)\|\psi(s, z)-\psi(s, v)\|_{2 p} d z d v\right)^{2} f^{2}(y) d y d s .
\end{aligned}
$$

By Lemma 4.1 and Proposition 3.4, we obtain

$$
\begin{aligned}
\left\|J_{1, \epsilon}\right\|_{p}^{2} & \leq c_{p}\|f\|_{L^{2}(\mathbb{R})}^{2} \int_{0}^{t}\left(\int_{\mathbb{R}} G_{t-s}(x-v) \int_{\mathbb{R}} G_{\epsilon}(v-z)|z-v|^{1 / 2} d z d v\right)^{2} d s \\
& \leq C \epsilon^{1 / 2} .
\end{aligned}
$$

For the term $J_{2, \epsilon}$ we can write, using Burkholder and Minkowski inequalities and applying Lemma 4.1

$$
\begin{aligned}
\left\|J_{2, \epsilon}\right\|_{p}^{2} \leq & c_{p} \int_{0}^{t} \int_{0}^{\infty}\left(\int_{\mathbb{R}} G_{t-s}(x-v)\left\|\psi_{\epsilon}^{-1}(s, v)\right\|_{2 p}\right. \\
& \left.\times \int_{\mathbb{R}} G_{\epsilon}(v-z)\left\|\frac{\partial \psi}{\partial z}(s, z)-\frac{\partial \psi}{\partial v}(s, v)\right\|_{2 p} d z d v\right)^{2} f^{2}(y) d y d s \\
\leq & c_{p}\|f\|_{L^{2}(\mathbb{R})}^{2} \int_{0}^{t}\left(\int_{\mathbb{R}^{2}} G_{t-s}(x-v) G_{\epsilon}(v-z)\left\|\frac{\partial \psi}{\partial z}(s, z)-\frac{\partial \psi}{\partial v}(s, v)\right\|_{2 p} d z d v\right)^{2} d s .
\end{aligned}
$$

By the continuity of $(s, z) \rightarrow \frac{\partial \psi}{\partial z}(s, z)$ in $L^{p}$, for any $p \geq 2$, in $[0, t] \times \mathbb{R}$, established in Remark 3.8, it follows that the integrand of the above integral on $[0, t]$ converges to zero for any $s \in[0, t]$. On the other hand, the integrand is bounded by an integrable function, which does not depend on $\epsilon$. Therefore, by the dominated convergence theorem, we conclude that $\left\|J_{2, \epsilon}\right\|_{p}^{2}$ converges to zero as $\epsilon$ tends to zero.

Finally for $J_{3, \epsilon}$, using Burkholder and Minkowski inequalities and applying Lemma 4.1, we have

$$
\begin{aligned}
\left\|J_{3, \epsilon}\right\|_{p}^{2} \leq & c_{p}\|f\|_{L^{2}(\mathbb{R})}^{2} \int_{0}^{t}\left(\int_{\mathbb{R}} G_{t-s}(x-v)\left\|\psi_{\epsilon}^{-2}(s, v)\right\|_{2 p}\right. \\
& \left.\times\left\|\psi(s, v) \frac{\partial \psi_{\epsilon}}{\partial v}(s, v)-\psi_{\epsilon}(s, v) \frac{\partial \psi}{\partial v}(s, v)\right\|_{2 p} d v\right)^{2} d s .
\end{aligned}
$$

For $(s, v) \in(0, t) \times \mathbb{R}$, the term $\left\|\psi(s, v) \frac{\partial \psi_{\epsilon}}{\partial v}(s, v)-\psi_{\epsilon}(s, v) \frac{\partial \psi}{\partial v}(s, v)\right\|_{2 p}$ converges to zero as $\epsilon$ tends to zero, due to the estimates (4.2) and (4.3). Therefore, by the dominated convergence theorem we conclude that $\left\|J_{3, \epsilon}\right\|_{p}^{2}$ tends to zero as $\epsilon$ tends to zero. The proof of (4.6) is similar and omitted. 
Step 4. Finally, we show that $A_{4, \epsilon}+A_{5, \epsilon}$ converges to zero in $L^{p}(\Omega)$ for all $p \geq 2$, as $\epsilon$ tends to zero. Once again, we show convergence of the terms when $z \geq y \geq 0$. When $z \leq y \leq 0$, the proof follows in the same way. The contribution of $\{y>0\}$ can be expressed as follows

$$
\begin{aligned}
H_{\epsilon}:=\int_{0}^{t} \int_{0}^{\infty} & \int_{\mathbb{R}} \frac{\partial}{\partial v} G_{t-s}(x-v) \frac{1}{\psi_{\epsilon}^{2}(s, v)}\left(\int_{y}^{\infty} G_{\epsilon}(v-z) \psi(s, z) d z\right) \\
& \times\left(\psi_{\epsilon}(s, v)-\int_{y}^{\infty} G_{\epsilon}(v-z) \psi(s, z) d z\right) \sigma_{s}(y)^{2} d v d y d s
\end{aligned}
$$

Adding and subtracting $\psi(s, v)$ in the second $d z$ integral, we get

$$
H_{\epsilon}=\sum_{i=1}^{3} H_{i, \epsilon}
$$

where

$$
H_{i, \epsilon}=\int_{0}^{t} \int_{0}^{\infty} \int_{\mathbb{R}} \frac{\partial}{\partial v} G_{t-s}(x-v) \frac{1}{\psi_{\epsilon}^{2}(s, v)}\left(\int_{y}^{\infty} G_{\epsilon}(v-z) \psi(s, z) d z\right) F_{i} \sigma_{s}(y)^{2} d v d y d s,
$$

where

$$
\begin{aligned}
& F_{1}:=\int_{-\infty}^{y} G_{\epsilon}(v-z) \psi_{\epsilon}(s, v) d z \\
& F_{2}:=\int_{y}^{\infty} G_{\epsilon}(v-z)\left[\psi_{\epsilon}(s, v)-\psi(s, v)\right] d z \\
& F_{3}:=\int_{y}^{\infty} G_{\epsilon}(v-z)[\psi(s, v)-\psi(s, z)] d z .
\end{aligned}
$$

We show convergence of each of these three terms, one at a time. To control the term $H_{1, \epsilon}$, apply Minkowski's inequality, Hölder's inequality, and Lemma 4.1, to get, for anu $p \geq 2$

$$
\left\|H_{1, \epsilon}\right\|_{p} \leq C \int_{0}^{t} \int_{0}^{\infty} \int_{\mathbb{R}} \frac{\partial}{\partial v} G_{t-s}(x-v)\left(\int_{y}^{\infty} G_{\epsilon}(v-z) d z\right)\left(\int_{-\infty}^{y} G_{\epsilon}(v-z) d z\right) f(y)^{2} d v d y d s .
$$

Notice that, for any fixed $s, y, v$, we have

$$
\frac{\partial}{\partial v} G_{t-s}(x-v)\left(\int_{y}^{\infty} G_{\epsilon}(v-z) d z\right)\left(\int_{-\infty}^{y} G_{\epsilon}(v-z) d z\right) f(y)^{2} \rightarrow 0
$$

as $\epsilon \rightarrow 0$. Furthermore, we can trivially bound this integrand by

$$
\left|\frac{\partial}{\partial v} G_{t-s}(x-v)\right| f(y)^{2}
$$

which is independent of $\epsilon$, and $(d v \otimes d y \otimes d s)$-integrable on $\mathbb{R} \times(0, \infty) \times[0, t]$. Hence, by dominated convergence, $\left\|H_{1, \epsilon}\right\|_{p} \rightarrow 0$ as $\epsilon \rightarrow 0$.

We bound the term with $H_{2, \epsilon}$ as follows

$$
\left\|H_{2, \epsilon}\right\|_{p} \leq C \sup _{s, v}\left\|\psi_{\epsilon}(s, v)-\psi(s, v)\right\|_{2 p} \int_{0}^{t} \int_{0}^{\infty} \int_{\mathbb{R}}\left|\frac{\partial}{\partial v} G_{t-s}(x-v)\right| f(y)^{2} d v d y d s
$$


for some positive constant $C>0$ and all $p \geq 2$. This quantity converges to zero as $\epsilon \rightarrow 0$ by Lemma 4.1. Lastly, apply the same techniques to get

$$
\begin{aligned}
\left\|H_{3, \epsilon}\right\|_{p} & \leq C \int_{0}^{t} \int_{0}^{\infty} \int_{\mathbb{R}}\left|\frac{\partial}{\partial v} G_{t-s}(x-v)\right|\left(\int_{y}^{\infty} G_{\epsilon}(v-z)|v-z|^{1 / 2} d z\right) f(y)^{2} d v d y d s \\
& \leq C \epsilon^{1 / 4} .
\end{aligned}
$$

which converges to zero as $\epsilon \rightarrow 0$. Therefore, $A_{4, \epsilon}+A_{5, \epsilon}$ converges to zero in $L^{p}(\Omega)$ as $\epsilon \rightarrow 0$, for all $p \geq 2$.

Step 5. As a conclusion, we deduce that the process $v(t, x)$ satisfies

$$
\begin{aligned}
v(t, x)=\int_{\mathbb{R}} G_{t}(x-y) u_{0}(y) d y & +\frac{1}{2} \int_{0}^{t} \int_{\mathbb{R}} \frac{\partial}{\partial y} G_{t-s}(x-y) v(s, y)^{2} d y d s \\
& +\int_{0}^{t} \int_{\mathbb{R}} G_{t-s}(x-y) \sigma_{s}(y) W(d s, d y) .
\end{aligned}
$$

Since $u$ also satisfies this equation, we have $u \equiv v$ by uniqueness of solutions.

\section{Regularity of Burgers' Equation}

We start with an easy, yet interesting, consequence of some of our results about $\psi$ and its regularity.

Proposition 5.1. Let $u(t, x)$ denote the solution to Burgers' equation (1.1). Assume that the initial condition $u_{0}$ is $\alpha$ Hölder continuous for some $\alpha \in(0,1)$. Then, for all $t, s \in[0, T]$, $x, y \in \mathbb{R}$, and $p \geq 2$, we have

$$
\|u(t, x)-u(s, y)\|_{p} \leq C\left(|t-s|^{\frac{\alpha}{2} \wedge\left(\frac{1}{4}-\frac{1}{2 q}\right)}+|x-y|^{\alpha \wedge\left(\frac{1}{2}-\frac{1}{q}\right)}\right) .
$$

Proof. Indeed, by adding and subtracting an appropriate term, we have

$$
\begin{aligned}
\|u(t, x)-u(t, y)\|_{p} & =2\left\|\frac{1}{\psi(s, y)} \frac{\partial \psi}{\partial y}(s, y)-\frac{1}{\psi(s, x)} \frac{\partial \psi}{\partial x}(s, x)\right\|_{p} \\
& \leq 2\left\|\frac{\partial \psi}{\partial y}(s, y) \frac{\psi(s, x)-\psi(s, y)}{\psi(s, x) \psi(s, y)}\right\|_{p}+2\left\|\frac{1}{\psi(s, x)}\left[\frac{\partial \psi}{\partial y}(s, y)-\frac{\partial \psi}{\partial x}(s, x)\right]\right\|_{p} \\
& \leq C\left(|x-y|^{1 / 2}+|x-y|^{\alpha \wedge\left(\frac{1}{2}-\frac{1}{q}\right)}\right),
\end{aligned}
$$

where the last inequality follows from Cauchy-Schwarz inequality, (3.3), and Propositions 3.4, 3.6, and 3.7. Using the same technique of adding and subtracting appropriate terms yields the desired regularity in $t$.

(i) From Remark 3.8 it follows that if we do not assume the Hölder continuity of $u_{0}$, then we have

$$
\|u(t, x)-u(s, y)\|_{p} \leq C(t \wedge s)^{-1 / 2}\left(|t-s|^{\frac{1}{4}-\frac{1}{2 q}}+|x-y|^{\frac{1}{2}-\frac{1}{q}}\right),
$$

Moreover, $u(t, x)$ is continuous in $L^{p}(\Omega)$ on $[0, T] \times \mathbb{R}$ for all $p \geq 2$.

(ii) Proposition (5.1) allows us to deduce the existence of a version of $u(t, x)$, which is locally Hölder continuous in space of order $\alpha \wedge\left(\frac{1}{2}-\frac{1}{q}\right)$ and in time of order $\frac{\alpha}{2} \wedge\left(\frac{1}{4}-\frac{1}{2 q}\right)$.

The next proposition provides some moment estimates for the solution to Burgers equation. 
Proposition 5.2. Let $u(t, x)$ denote the solution to Burgers' equation (1.1) Then, for all $t \in[0, T]$ and $x \in \mathbb{R}$, and $p \geq 2$, we have

$$
\sup _{x \in \mathbb{R}}\|u(t, x)\|_{p} \leq K c_{2 p}(t \vee 1)^{1-\frac{1}{q}} \exp \left(\|f\|_{L^{2}(\mathbb{R})}^{2} t\left(1 / 8+c_{2 p}+\|f\|_{L^{2}(\mathbb{R})}^{2} t / 2\right)+\frac{1}{4 p}\left\|u_{0}\right\|_{L^{1}(\mathbb{R})}\right),
$$

where $c_{p}$ is the optimal constant in Burkholder's inequality and $K$ is a constant depending on $q,\|f\|_{L^{q}(\mathbb{R})},\|f\|_{L^{2}(\mathbb{R})},\left\|u_{0}\right\|_{L^{1}(\mathbb{R})}$, and $\left\|u_{0}\right\|_{\infty}$.

Proof. By Hölder's inequality, we can write

$$
\|u(t, x)\|_{p}=2\left\|\psi(t, x)^{-1} \frac{\partial \psi}{\partial x}(t, x)\right\|_{p} \leq 2\left\|\psi(t, x)^{-1}\right\|_{2 p}\left\|\frac{\partial \psi}{\partial x}(t, x)\right\|_{2 p} .
$$

Then, the result follows from Remark 3.2 and Proposition 3.6.

\section{REFERENCES}

[1] L. Bertini, N. Cancrini, G. Jona-Lasinio, The Stochastic Burgers Equation, Commun. Math. Phys. 165, 211-232 (1994)

[2] L. Chen and R. Dalang, Moments and Growth Indices for the Nonlinear Stochastic Heat Equation with Rough Initial Conditions, Ann. Probab. 43, 3006-3051 (2015)

[3] I. Gyöngy and D. Nualart, On the Stochastic Burgers Equation in the Real Line, Ann. Probab. 27, $782-802(1999)$

[4] J. Leòn, D. Nualart, and R. Pettersson, The Stochastic Burgers Equation: Finite Moments and Smoothness of the Density, Infinite Dimensional Analysis 3, 363-385 (2000)

[5] J. Walsh, An Introduction to Stochastic Partial Differential Equations, Ècole d'Été de Probabilités de Saint-Flour, XIV-1984, Lecture Notes in Math., 1180, Springer, Berlin, 265-439 (1986)

Department of Mathematics, University of Kansas, Lawrence, KS 66045, USA

E-mail address: plewis85@ku.edu

E-mail address: nualart@ku.edu 